\title{
The MOSDEF Survey: No Significant Enhancement in Star Formation or Deficit in Metallicity in Merging Galaxy Pairs at $1.5 \lesssim z \lesssim 3.5^{*}$
}

\author{
Tim J. Wilson ${ }^{1}$, Alice E. Shapley ${ }^{2}$ (1), Ryan L. Sanders ${ }^{2}$ (1), Naveen A. Reddy ${ }^{3}$ (D), William R. Freeman ${ }^{3}$ (1), Mariska Kriek ${ }^{4}$ (1), \\ Irene Shivaei ${ }^{5}$ (1), Alison L. Coil ${ }^{6}$ (1), Brian Siana ${ }^{3}$ (1), Bahram Mobasher ${ }^{3}$, Sedona H. Price ${ }^{7}$ (i), Mojegan Azadi ${ }^{8}$ (1) \\ Guillermo Barro ${ }^{9}$ (i), Laura de Groot $^{10}$ (ID), Tara Fetherolf ${ }^{3}$, Francesca M. Fornasini ${ }^{8}$ (1D, Gene C. K. Leung ${ }^{6}$ (D), and Tom O. Zick ${ }^{4}$ \\ ${ }^{1}$ Department of Physics, University of California, Berkeley, 366 LeConte Hall MC 7300, Berkeley, CA 94720, USA; tim.wilson@berkeley.edu \\ ${ }_{3}^{2}$ Department of Physics and Astronomy, University of California, Los Angeles, 430 Portola Plaza, Los Angeles, CA 90095, USA \\ ${ }^{3}$ Department of Physics and Astronomy, University of California, Riverside, 900 University Avenue, Riverside, CA 92521, USA \\ ${ }_{4}^{4}$ Astronomy Department, University of California at Berkeley, Berkeley, CA 94720, USA \\ ${ }^{5}$ Steward Observatory, University of Arizona, 933 N Cherry Avenue, Tucson, AZ 85721, USA \\ ${ }^{6}$ Center for Astrophysics and Space Sciences, Department of Physics, University of California, San Diego, 9500 Gilman Drive, La Jolla, CA 92093, USA \\ ${ }_{8}^{7}$ Max-Planck-Institut für Extraterrestrische Physik, Postfach 1312, Garching, D-85741, Germany \\ ${ }^{8}$ Harvard-Smithsonian Center for Astrophysics, 60 Garden Street, Cambridge, MA 02138, USA \\ ${ }^{9}$ Department of Physics, University of the Pacific, 3601 Pacific Avenue, Stockton, CA 95211, USA \\ ${ }^{10}$ Department of Physics, The College of Wooster, 1189 Beall Avenue, Wooster, OH 44691, USA \\ Received 2018 August 29; revised 2019 February 4; accepted 2019 February 12; published 2019 March 18
}

\begin{abstract}
We study the properties of 30 spectroscopically identified pairs of galaxies observed during the peak epoch of star formation in the universe. These systems are drawn from the MOSFIRE Deep Evolution Field (MOSDEF) Survey at $1.4 \leqslant z \leqslant 3.8$, and are interpreted as early-stage galaxy mergers. Galaxy pairs in our sample are identified as two objects whose spectra were collected on the same Keck/MOSFIRE spectroscopic slit. Accordingly, all pairs in the sample have projected separations $R_{\text {proj }} \leqslant 60 \mathrm{kpc}$. The velocity separation for pairs was required to be $\Delta v \leqslant 500 \mathrm{~km} \mathrm{~s}^{-1}$, which is a standard threshold for defining interacting galaxy pairs at low redshift. Stellar mass ratios in our sample range from 1.1 to 550, with 12 ratios closer than or equal to 3:1, the common definition of a "major merger." Studies of merging pairs in the local universe indicate an enhancement in star formation activity and deficit in gas-phase oxygen abundance relative to isolated galaxies of the same mass. We compare the MOSDEF pairs sample to a control sample of isolated galaxies at the same redshift, finding no measurable SFR enhancement or metallicity deficit at fixed stellar mass for the pairs sample. The lack of significant difference between the average properties of pairs and control samples appears in contrast to results from low-redshift studies, although the small sample size and lower signal-to-noise of the high-redshift data limit definitive conclusions on redshift evolution. These results are consistent with some theoretical works, suggesting a reduced differential effect of precoalescence mergers on galaxy properties at high redshift—specifically that precoalescence mergers do not drive strong starbursts.
\end{abstract}

Key words: galaxies: evolution - galaxies: high-redshift - galaxies: interactions

\section{Introduction}

Galaxies grow in mass through a combination of mergers with other galaxies and smooth accretion of baryons and dark matter. Predicting the frequency of both major (i.e., with roughly equal masses) and minor (i.e., with significantly unequal masses) mergers as a function of galaxy mass and redshift is therefore an important component of hierarchical models of structure formation (e.g., Hopkins et al. 2010). At the same time, obtaining empirical constraints on such merger rates as a function of galaxy mass and redshift represents a key goal for observations of galaxy evolution (e.g., Lotz et al. 2011). In addition to quantifying merger rates, both models and observations aim to describe the impact of galaxy interactions on the properties of merging and coalesced galaxies.

Simulations of star-forming galaxy mergers predict a characteristic progression of the star formation rate (SFR) throughout the merger event. Relative to the time prior to the

\footnotetext{
* Based on data obtained at the W.M. Keck Observatory, which is operated as a scientific partnership among the California Institute of Technology, the University of California, and the National Aeronautics and Space Administration, and was made possible by the generous financial support of the W.M. Keck Foundation.
}

merger, the SFRs of the merging galaxies are elevated during their extended gravitational interaction, and ultimately peak when the galaxies coalesce (e.g., Mihos \& Hernquist 1996; Cox et al. 2008; Hopkins et al. 2008). The degree of enhancement in SFR is predicted to depend on galaxy mass ratio. For example, Cox et al. (2008) has demonstrated that mergers with mass ratio smaller than 3:1 lead to much stronger bursts of star formation than mergers with larger mass ratios. Additional factors affect the strength of the merger-induced starburst, such as the orientation of the orbits of merging galaxies, as well as their structural properties and gas fractions.

In the local universe, the most luminous systems, (i.e., ultraluminous infrared galaxies; ULIRGs) appear to be dominated by advanced-stage major mergers during or just after coalescence (e.g., Sanders \& Mirabel 1996; Tacconi et al. 2002). Precoalescence stages of merging at $z \sim 0$ have been traced by galaxy pairs. The Sloan Digital Sky Survey (SDSS) has yielded a statistical sample of such pairs (e.g., Ellison et al. 2008b; Patton et al. 2011, 2013; Scudder et al. 2012, 2015), identified as galaxies separated by both a small projected radius (with upper limits on $R_{\text {proj }}$ ranging from 30 to $80 \mathrm{kpc}$ ) and small radial velocity difference (with upper limits on $\Delta v$ ranging from 200 to $500 \mathrm{~km} \mathrm{~s}^{-1}$ ). Members of these galaxy 
pairs are characterized by both enhanced SFRs (e.g., $\sim 60 \%$ out to $30 \mathrm{kpc}$; Scudder et al. 2012) and depressed gas-phase oxygen abundances (e.g., 0.02 dex; Scudder et al. 2012) relative to a control sample of isolated galaxies matched in stellar mass. Such differences are consistent with theoretical models of galaxy mergers in which an increase in SFR accompanies the inflow of gas into the central regions of the merging galaxies, which also tends to dilute the metal content of the interstellar medium (ISM; Hopkins et al. 2008; Bustamante et al. 2018). A similar enhancement in SFR in galaxy pairs has been detected out to $z \sim 1$ (Lin et al. 2007; Wong et al. 2011).

Galaxy mergers have now been identified out to $z \sim 6$ (Ventou et al. 2017). In the early universe (i.e., at $z>1$ ), primarily one of two techniques is employed to flag merging systems. First, it is common to use morphological signatures to identify ongoing or recently completed merger events. Galaxies have been visually classified as mergers on the basis of morphological features, such as tidal tails and bridges, and double nuclei (Lofthouse et al. 2017) and also identified as interacting based on nonparametric morphological statistics, such as the Gini and $M_{20}$ coefficients (Lotz et al. 2004), or the concentration (C), asymmetry (A), and clumpiness (S) statistics (Conselice 2014). The second technique for flagging mergers is through galaxy pairs. Many studies aiming to quantify the merger fraction and rate at $z>1$ have been based on photometric pairs, which consist of galaxies within a small projected radius and small difference in photometric redshift (e.g., Williams et al. 2011; Man et al. 2012, 2016; Mantha et al. 2018). In some cases (Bluck et al. 2009), the photometric redshift for only one of the galaxies is known. The possibility of contamination by chance projections must therefore be accounted for, especially when the redshift of a potential companion galaxy is unknown. Recently, merging pairs at $z>1$ have also been identified spectroscopically, based on rest-frame ultraviolet spectra (Tasca et al. 2014; Ventou et al. 2017). However, the sensitivity of rest-frame UV features to large-scale galaxy outflows (Pettini et al. 2001; Shapley et al. 2003; Steidel et al. 2010) limits the accuracy with which such galaxy systemic redshifts, and therefore merger dynamics, can be measured.

To date, most studies of merging pairs at $z>1$ have focused on global statistics, such as the merger fraction and rate, as opposed to systematic studies of the impact of close interactions on the properties of merging galaxies. In this work, we focus on the latter, based on a sample of galaxy mergers identified at $1.4 \leqslant z \leqslant 3.8$ within the MOSFIRE Deep Evolution Field (MOSDEF) survey (Kriek et al. 2015). The extensive rest-optical spectroscopic coverage of the MOSDEF survey enables us to assemble a clean sample of galaxy pairs that are not only close on the sky but also in redshift space. With spectroscopic pairs, there is little possibility of contamination by chance projections of completely unassociated galaxies. Such chance projections can arise when pairs are identified on the basis of photometric redshifts, given their associated uncertainties at high redshift. ${ }^{11}$ Furthermore, we have estimated key galaxy properties such as SFR, stellar mass

\footnotetext{
${ }^{11}$ We note that proximity in redshift space does not guarantee merging, as galaxy pairs offset by tens of proper kpc in $R_{\text {proj }}$ and with line of sight velocity separations of up a few hundreds of $\mathrm{km} \mathrm{s}^{-1}$ may not be bound and destined to merge (Moreno et al. 2013). However, with spectroscopic redshift measurements, we can at least apply the same proximity criteria in velocity space that is used for studies of local galaxy pairs.
}

$\left(M_{*}\right)$, and gas-phase oxygen abundance for both merging and isolated systems and can therefore study for the first time the effect of interactions on star formation activity and chemical enrichment in distant star-forming galaxies.

In Section 2, we present the details of the MOSDEF survey and the galaxy properties analyzed in this work. Section 3 discusses the selection and properties of spectroscopically determined merging pairs in MOSDEF, while Section 4 describes the selection of our control sample of isolated galaxies used for systematic comparison with mergers. In Section 5, we investigate the effect of mergers on star formation and metal enrichment through analysis of the SFR- $M_{*}$ main sequence and stellar massmetallicity relation (MZR) for both merging pairs and isolated control galaxies. We present a discussion of our results and describe future work in Section 6. Throughout this paper, we adopt cosmological parameters of $H_{0}=70 \mathrm{~km} \mathrm{~s}^{-1} \mathrm{Mpc}^{-1}$, $\Omega_{M}=0.30$, and $\Omega_{\Lambda}=0.7$.

\section{The MOSDEF Survey}

We assembled a sample of spectroscopically confirmed merging pairs from the MOSDEF survey. With MOSDEF, we performed a large survey of the rest-frame optical spectra of $\sim 1500$ galaxies spanning $1.4 \leqslant z \leqslant 3.8$. Spectra were collected for MOSDEF galaxy targets using the MOSFIRE spectrograph (McLean et al. 2012) on the Keck I telescope. For a full description of the MOSDEF survey observations and data reduction, we refer readers to Kriek et al. (2015). Here, we provide the survey information most relevant to the current work.

MOSDEF observing runs comprised 48.5 MOSFIRE nights between 2012 December and 2016 May. Galaxies in the MOSDEF sample are concentrated in three redshift intervals where strong rest-optical emission lines fall within windows of atmospheric transmission $(1.37 \leqslant z \leqslant 1.70,2.09 \leqslant z \leqslant 2.61$, and $2.95 \leqslant z \leqslant 3.80$ ). The targeted galaxies were selected from the photometric and spectroscopic catalogs constructed as part of the 3D-HST survey (Brammer et al. 2012; Skelton et al. 2014; Momcheva et al. 2016) down to limiting HST/WFC3 F160W AB magnitudes of 24.0, 24.5, and 25.0, respectively at $1.37 \leqslant z \leqslant 1.70,2.09 \leqslant z \leqslant 2.61$, and $2.95 \leqslant z \leqslant 3.80$. As these galaxies are primarily located in three CANDELS fields: AEGIS, COSMOS, and GOODSN (Grogin et al. 2011; Koekemoer et al. 2011), they have extensive multi-wavelength photometric coverage (Skelton et al. 2014) from which stellar population parameters and photometric redshifts are derived.

Targets at $1.37 \leqslant z \leqslant 1.70$ were typically observed using $1 \mathrm{hr}$ exposures in the $Y, J$, and $H$ bands; those at $2.09 \leqslant$ $z \leqslant 2.61$ were observed using $2 \mathrm{hr}$ exposures in the $J, H$, and $K$ bands. Those at $2.95 \leqslant z \leqslant 3.80$ were observed using $2 \mathrm{hr}$ exposures in the $H$ and $K$ bands. Our MOSFIRE multi-object slit masks typically contained $\sim 30 \quad 0$. 7 slits, yielding a resolution of 3400 in $Y, 3300$ in $J, 3650$ and $H$, and 3600 in $K$. In practice, galaxy pairs are identified in this work on the basis of the strongest rest-frame optical features, which are $\mathrm{H} \alpha$ and [O III $] \lambda 5007 . \mathrm{H} \alpha$ is measured in the $H$ and $K$ bands, respectively, at $1.37 \leqslant z \leqslant 1.70$ and $1.90 \leqslant z \leqslant 2.61$, while [O III] $\lambda 5007$ is measured in the $J$ and $H$ bands, respectively, over the same redshift ranges and in the $K$ band at $2.95 \leqslant z \leqslant 3.80$.

We used a custom IDL pipeline to reduce the raw data and produce two-dimensional spectra in each filter, as described in Kriek et al. (2015). One-dimensional science and error spectra 
were then optimally extracted from the two-dimensional spectra (Freeman et al. 2017). The final MOSDEF sample contains 1493 primary targets, 66 of which represent duplicate observations. In addition, the sample includes 165 galaxies that serendipitously fell within MOSDEF slits and for which we measured spectroscopic redshifts (hereafter serendips).

Due to the nature of our MOSFIRE slit observations and the manner in which serendips were identified, special care is required to obtain accurate flux and wavelength information for serendips. The coordinates of MOSDEF primary targets determined the location of each MOSFIRE slit, and therefore primary targets are well centered in the slits. We identify galaxy pairs by the presence of a serendip companion galaxy, whose light also falls in the slit of the primary target and whose spectrum yields a redshift close to that of the primary target. As the position of the serendip was not taken into account when designing MOSDEF slit masks, such galaxies are not necessarily centered across the slits that capture their light. Therefore, when we apply slit-loss corrections to the spectra for each galaxy in each filter (Kriek et al. 2015), we take into account the potentially off-center nature of serendip sources. The potential horizontal offset of a serendip also leads to a small offset in the actual wavelength solution that should be calculated for the serendip relative to what is derived based on sky lines that fill and are centered in the slit. However, the velocity offsets corresponding to spatial offsets of less than or equal to half of a slit width are small $\left(\leqslant 50 \mathrm{~km} \mathrm{~s}^{-1}\right)$ compared to the range of velocity offsets between primary and serendip objects considered in this work and do not affect any of our conclusions. Therefore, we do not correct for such offsets.

We measured emission-line fluxes by fitting Gaussian profiles to one-dimensional spectra. The MOSFIRE redshift for each galaxy, $z_{\text {MOSFIRE}}$, was estimated from the centroid of the highest $\mathrm{S} / \mathrm{N}$ feature detected (i.e., typically $\mathrm{H} \alpha$ or [O III] 15007). Balmer emission-line fluxes were corrected for underlying stellar absorption based on the best-fit stellar population model to the observed broadband spectral energy distribution (SED). Several galaxy properties were derived for our targets based on MOSFIRE emission-line fluxes and existing multi-wavelength imaging data. These include the nebular extinction, $E(B-V)_{\text {neb }}$, based on the observed $\mathrm{H} \alpha / \mathrm{H} \beta$ Balmer decrement and assuming a Milky Way dust extinction curve (Cardelli et al. 1989). $\mathrm{H} \alpha$ SFRs (SFR(H $\alpha)$ ) were then estimated from the dust-corrected $\mathrm{H} \alpha$ luminosities, based on the Hao et al. (2011) update to the calibration of Kennicutt (1998) and assuming a Chabrier (2003) IMF. Stellar masses $\left(M_{*}\right)$ were estimated by using the fitting program, FAST (Kriek et al. 2009), to fit the stellar population synthesis models of Conroy et al. (2009) to galaxy broadband photometric SEDs, assuming a Chabrier (2003) IMF, solar metallicity, and a Calzetti et al. (2000) dust attenuation law. We also assumed delayed exponential star formation histories of the form SFR $\propto$ $t \exp (-t / \tau)$, with $t$ the time since star formation commenced and $\tau$ the star formation decay timescale. Accordingly, SED fitting also yielded an independent estimate of the SFR; i.e., SFR(SED). Finally, gas-phase oxygen abundances were estimated from two empirical calibrations commonly applied at high redshift. We used the calibrations of Pettini \& Pagel (2004) based on the N2 and O3N2 emission-line indicators, which are defined as, $\mathrm{N} 2=[\mathrm{N} \mathrm{II}] \lambda 6584 / \mathrm{H} \alpha$ and $\mathrm{O} 3 \mathrm{~N} 2=$
$([\mathrm{O}$ III $] \lambda 5007 / \mathrm{H} \beta) /([\mathrm{N}$ II $] / \mathrm{H} \alpha)$. These calibrations are:

$$
12+\log (\mathrm{O} / \mathrm{H})_{\mathrm{N} 2}=8.90+0.57 \times \log (\mathrm{N} 2)
$$

and

$$
12+\log (\mathrm{O} / \mathrm{H})_{\mathrm{O} 3 \mathrm{~N} 2}=8.73+0.32 \times \log (\mathrm{O} 3 \mathrm{~N} 2) .
$$

Although there has been considerable debate in the literature regarding the validity of these locally calibrated metallicity indicators in an absolute sense for high-redshift galaxies (e.g., Steidel et al. 2014; Sanders et al. 2015), these indicators should be adequate for estimating relative metallicity differences among high-redshift galaxies.

\section{Merging Pair Selection}

In order to identify spectroscopic merging pairs within MOSDEF, we applied the following criteria, which are broadly motivated by the low-redshift study of Ellison et al. (2008b):

1. The spectra of two or more galaxies must have been collected in a single MOSDEF spectroscopic slit, comprising the primary galaxy target and at least one serendip. This criterion effectively translates into a cut on

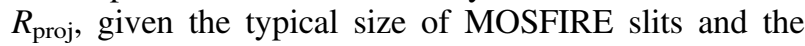
small variation in angular size for a fixed proper distance over the redshift range of our sample.

2. The primary and serendip galaxies must both have secure MOSFIRE spectroscopic redshifts.

3. The two objects must be separated by $\Delta v=c \mid z_{\text {primary }}-$ $z_{\text {serendip }} / /\left(1+z_{\text {primary }}\right) \leqslant 500 \mathrm{~km} \mathrm{~s}^{-1}$, where $z_{\text {primary }}$ is the redshift of the primary target and $z_{\text {serendip }}$ is the redshift of the serendip.

Adopting the above criteria, we have spectroscopically identified 31 merging pairs, one of which was observed twice (with primary and serendip classifications reversed). Given the small sample size, we carefully inspected each pair in both twodimensional spectra and HST F160W images to confirm the validity of our pair identifications. In particular, for pairs with

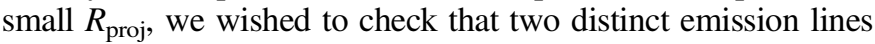
could be ascertained in the two-dimensional spectra and that two distinct brightness concentrations could be determined in the HST images, corresponding to the separate 3D-HST catalog identifiers. This close analysis caused us to remove one apparent pair from our initial sample, as the HST image revealed the serendip object to be a single bright knot within the more extended light distribution of the primary. Furthermore, the twodimensional MOSFIRE spectrum showed an extended, tilted emission line that did not clearly break up into two components. Accordingly, our final sample consists of 30 dynamical pairs.

One of the 30 serendips (primary target COSMOS-25229, $z=2.1813$ ) was clearly apparent in the HST F160W image but had no identifier or multi-wavelength SED in the 3D-HST photometric catalog (Skelton et al. 2014). We include the corresponding pair in our analysis, as the properties of the primary target can still be considered differentially with those of our control sample described in the next section. We also note that 15 of the serendips were contained within the MOSDEF parent catalog and could have been targeted for spectroscopy as part of the MOSDEF survey, whereas 15 were fainter than the MOSDEF limits for targeted spectroscopy or had photometric redshifts outside the MOSDEF target redshift ranges. There is 

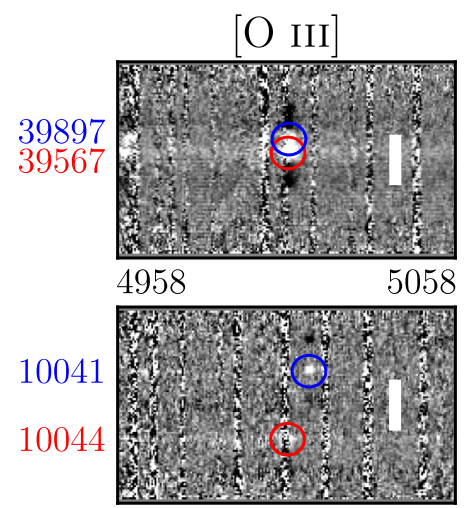

$$
4958
$$

5058
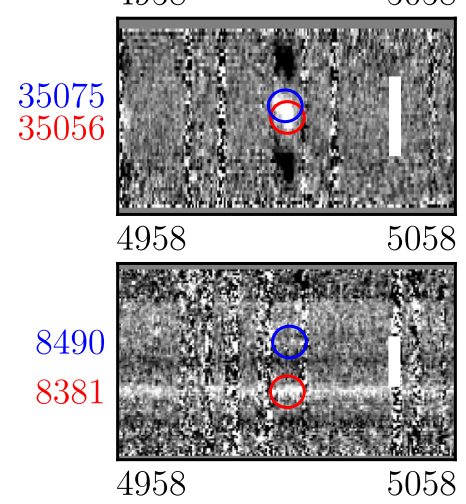

5058
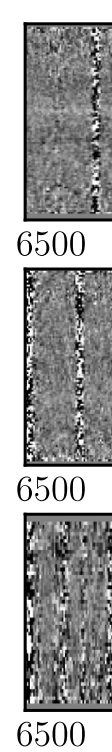

$\mathrm{H} \alpha$

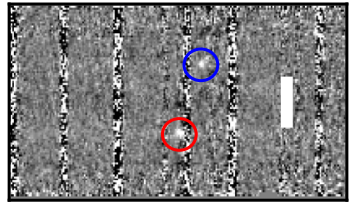

6500

6630

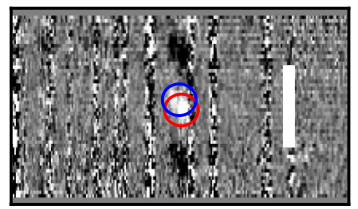

Fil

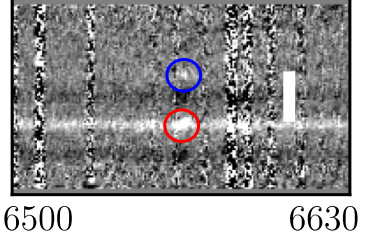

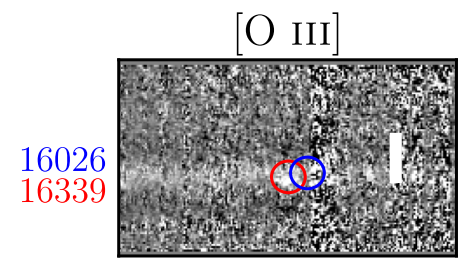

$$
4958
$$

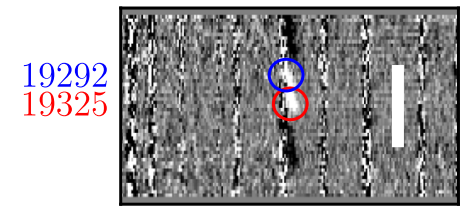

4958

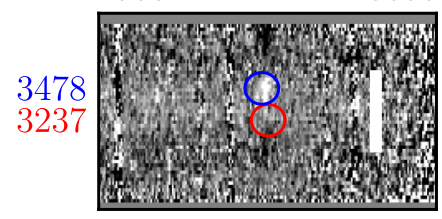

4958
5058
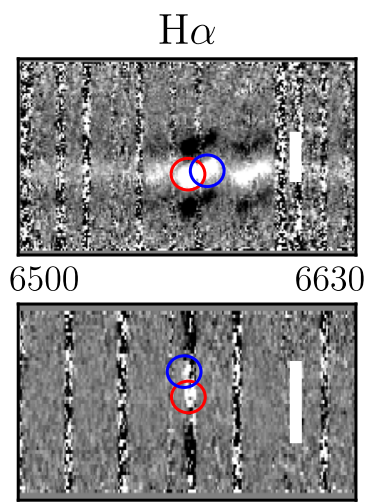

6500

6630

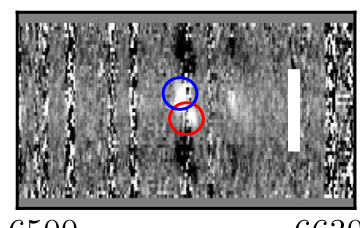

6500

6630

Rest Wavelength $(\AA)$

\section{Rest Wavelength $(\AA)$}

Figure 1. Gallery of two-dimensional spectra of galaxy pairs at $1.37 \leqslant z \leqslant 1.70$. Two panels are shown for each galaxy pair, which zoom in on the rest-frame wavelength ranges centered on [O III] $\lambda 5007$ and $\mathrm{H} \alpha$ for the primary target, and correspond to $\Delta z= \pm 0.01$. At these redshifts, [O III] $\lambda 5007$ falls in the observed $J$ band, while $\mathrm{H} \alpha$ falls in the observed $H$ band. For each pair, emission from the primary target galaxy is circled in red, while that from the serendipitous companion is circled in blue. 3D-HST v4.1 catalog numbers (Skelton et al. 2014) are given to the left of the [O III] $\lambda 5007$ panel, with red and blue color-coding corresponding to the circles. Spectral cut-outs are scaled to the same vertical size on the page for display purposes, resulting in a variable angular scale in that dimension. Accordingly, we provide a white vertical scale bar in each panel indicating the extent of 30 proper kpc, which corresponds to 3 ." 65 at the median redshift of the sample.

also one case in which two associated serendips were identified on the slit along with the primary target (i.e., primary GOODSN23344, $z=2.4839$ ). Accordingly, our 30 pairs comprise 29 primary galaxies and 30 serendips for a total of 59 galaxies.

Galleries of two-dimensional [O III] and $\mathrm{H} \alpha$ emission-line spectra indicating primary and serendip objects are contained in Figures 1-3. For the subsample of $z \sim 3$ pairs, we only show [O III] because these pairs lack $\mathrm{H} \alpha$ coverage. For pairs at $z \leqslant 2.7$ with both $[\mathrm{O} \mathrm{III}]$ and $\mathrm{H} \alpha$ coverage, it can be seen that the two-dimensional emission-line morphologies are typically very similar in [O III] and $\mathrm{H} \alpha$. Key properties of our galaxy pairs are summarized in Tables 1 (redshift, apparent magnitude, projected physical separation, and line of sight velocity separation) and 2 (stellar mass, SFR, and metallicity). In Figure 4, we show example HST F160W postage stamps indicating the range of properties in our sample: a widely separated pair; a triple of associated galaxies; and a small-separation pair that is apparently close to coalescence.

The 30 pairs in our sample span the redshift interval $1.4 \leqslant z \leqslant 3.5$. Since the pairs at $z>2.65$ lack coverage of $\mathrm{H} \alpha$ emission, we are unable to determine robust Balmer decrements and therefore dust-corrected SFRs based on Balmer emission lines. For these six $z \sim 3$ pairs, we also do not have access to the N2 and O3N2 metallicity indicators (Pettini \& Pagel 2004), which are commonly applied at $z \leqslant 2.65$. Accordingly, we list the basic parameters for the $z \sim 3$ pairs but do not analyze their differential physical properties in the spaces of SFR, mass, and metallicity in Section 5. Our differential analysis focuses on the 24 remaining pairs at $1.4 \leqslant z \leqslant 2.6$. This redshift range overlaps the epoch of peak star formation in the history of the universe (Madau \& Dickinson 2014). Therefore, our study enables us to trace the impact of early-stage mergers on galaxy properties during its most active period. To maximize our sample size, we analyze all 24 pairs together. Although the sample spans a significant range in cosmic time $(1.9 \mathrm{Gyr})$, we justify the joint analysis across this redshift interval based on the results of Shivaei et al. (2015), who demonstrated that there is no significant evolution in the star-forming main sequence (i.e., the SFR versus stellar mass relation) between $z \sim 1.4$ and 2.6 .

One of the unique features of our sample is the precise spectroscopic redshifts available for our targets. We use these redshifts to calculate the velocity separations between primary objects and serendips, and adopt an upper limit for pair velocity separations of $\Delta v=500 \mathrm{~km} \mathrm{~s}^{-1}$. This limit is chosen to match the one adopted in Ellison et al. (2008b) for analysis of merging pairs in the local universe. We cannot establish definitively that the spectroscopic pairs we have identified will merge. However, simulations show that over this range of velocity separations, the majority of pairs are bound and will eventually coalesce (e.g., Moreno et al. 2013). The distribution of line of sight velocity separations is shown in Figure 5(a).

We also calculated the projected physical separation, $R_{\text {proj }}$, between primary and serendip objects using their mean redshift 

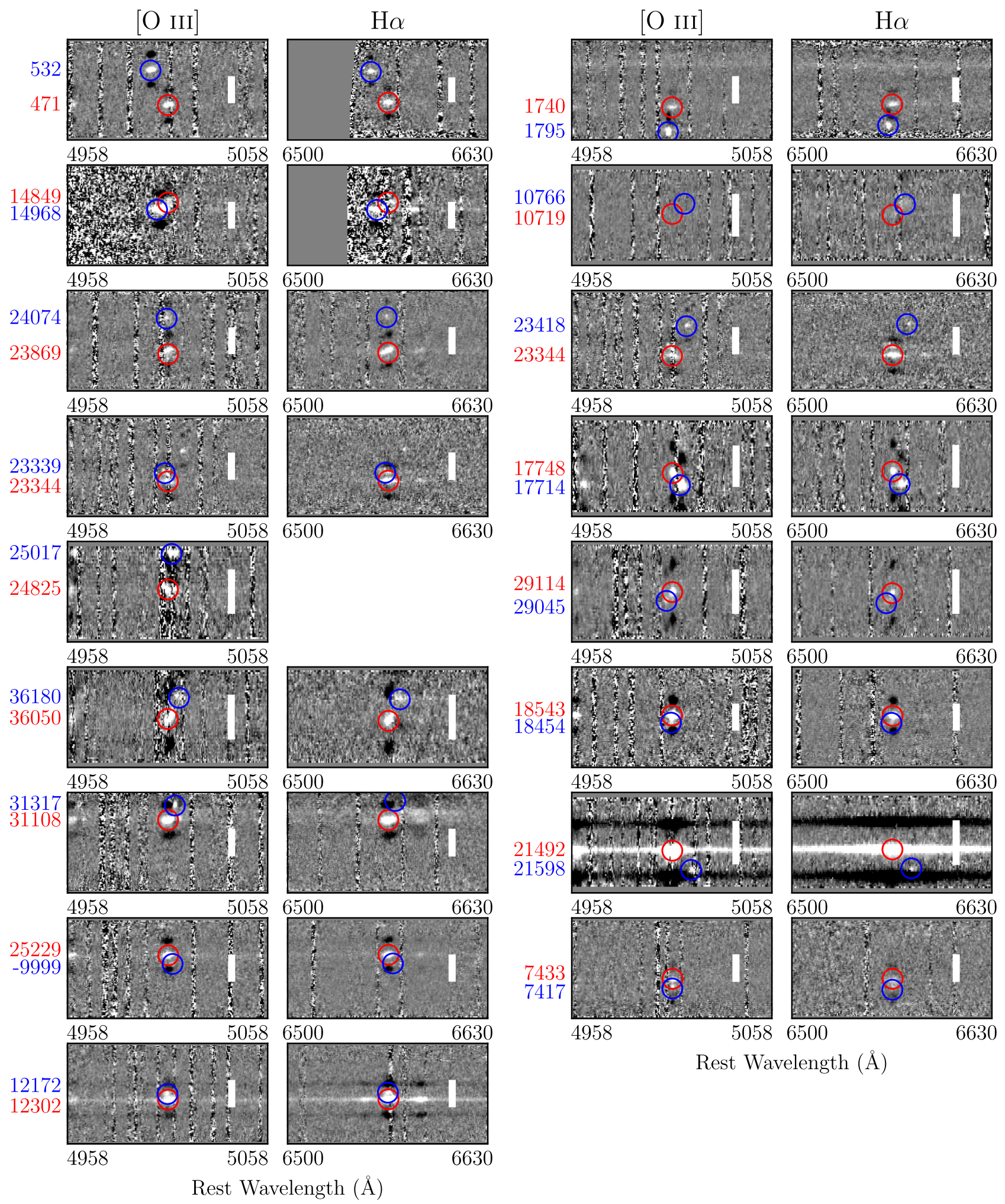

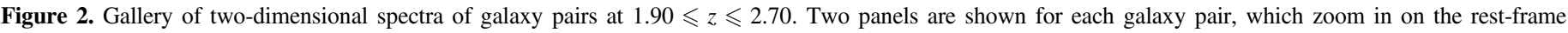

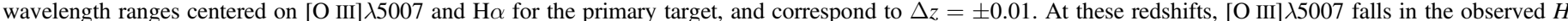

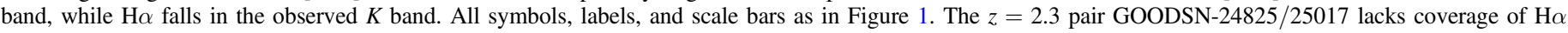
because it was observed as a filler target on a MOSDEF mask targeting $1.37 \leqslant z \leqslant 1.70$ galaxies and therefore not observed in the $K$ band.

and sky coordinates in the 3D-HST catalog (Skelton et al. 2014). In the one case of the serendip unidentified in the 3D$H S T$ catalog, we measured its position directly from the $H S T$ F160W image. The distribution in $R_{\text {proj }}$ is shown in Figure 5(b). In part due to the typical MOSFIRE slit length of 7 !" 1 , all of the projected physical separations in our sample are smaller than $60 \mathrm{kpc}$, which is well within the limit of $80 \mathrm{kpc}$ adopted by Ellison et al. (2008b). As another basic pair parameter, we estimated the stellar mass ratio for each pair, normalized in each case as the ratio between the mass of the more massive 

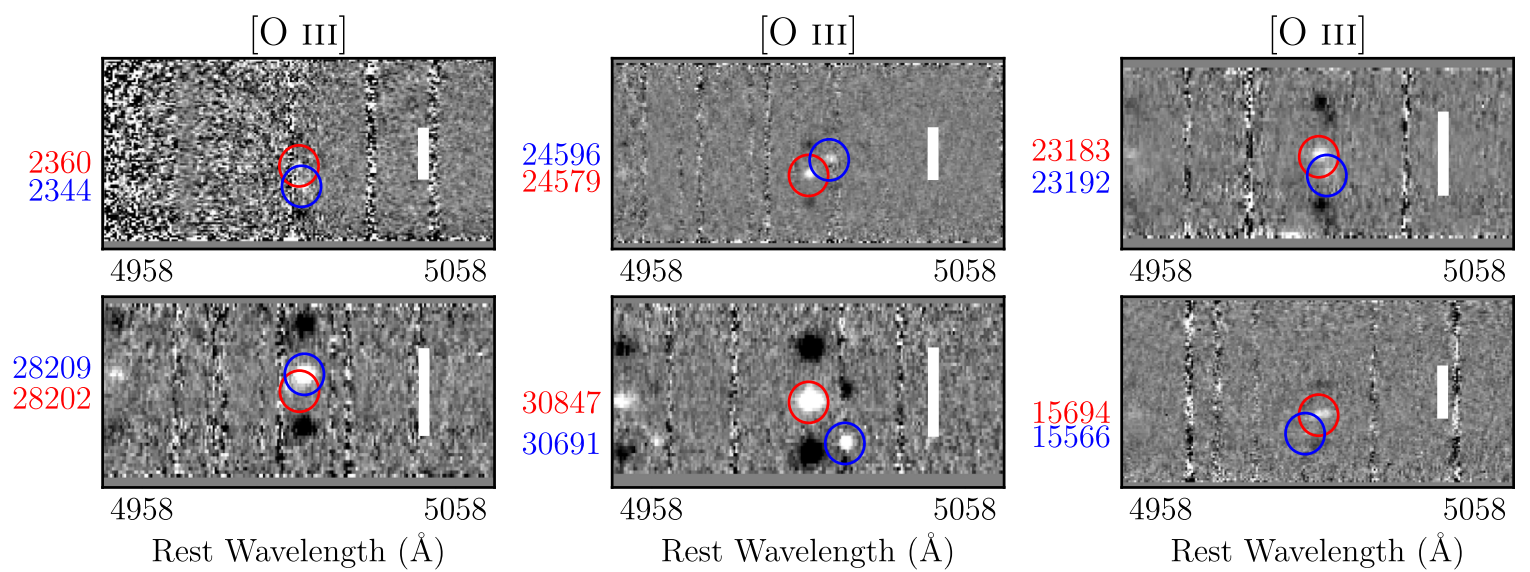

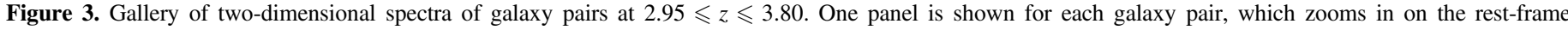

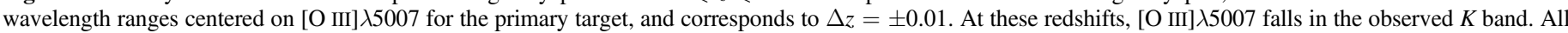
symbols, labels, and scale bars as in Figure 1.

galaxy to that of the less massive galaxy (as opposed to the primary-to-serendip mass ratio). The distribution of mass ratios in our sample is shown in Figure 5(c). This distribution spans from 1.1 up to 550, but all except one of the pairs have mass ratios that fall between 1.1 and 17 (this smaller range in mass ratio is shown in Figure 5(c), for improved display quality). Out of our pair sample, 12 have mass ratios of 3:1 or smaller, which would cause them to be classified as major mergers (Cox et al. 2008).

One potential limitation of our data set of merging pairs is its incompleteness due to the small fraction of on-sky area surrounding each primary galaxy that is sampled by the MOSFIRE spectroscopic slit. Over the range of redshifts of our pairs sample, the typical MOSFIRE slit dimensions of 7 !! $1 \times 0$ !" 7 subtend only $\sim 3 \%$ of the on-sky area out to a projected physical radius of $60 \mathrm{kpc}$. The small fractional area subtended by the MOSFIRE slit may have caused us to miss companion galaxies that are actually closer to the primary galaxy in projected radius than the companions we identifiedand therefore more relevant from the standpoint of merger interactions-but do not happen to fall inside the MOSFIRE slit.

To assess the importance of this effect, we searched within a projected radius of $60 \mathrm{kpc}$ of each primary galaxy for any additional galaxies with spectroscopic redshifts placing them within $500 \mathrm{~km} \mathrm{~s}^{-1}$ of the primary galaxy's spectroscopic redshift. If no spectroscopic redshift was available for galaxies within $60 \mathrm{kpc}$, we considered the "grism" redshifts from the 3D-HST catalogs based on a combined fit to the broadband

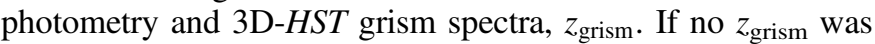
available, we used the $3 \mathrm{D}-H S T$ photometric redshift, $z_{\text {phot }}$, determined from the broadband photometry (Momcheva et al. 2016). For neighboring galaxies with only grism or photometric redshifts, we identified a galaxy as a potential companion if the $68 \%$ confidence interval of its redshift probability distribution encompassed the spectroscopic redshift of the primary galaxy. According to these criteria, we found that 21 out of 29 primary galaxies in our sample had additional potential galaxy companions within a projected radius of $60 \mathrm{kpc}$, including only a single new companion that was spectroscopically confirmed. The remaining potential companion galaxies had only photometric or grism redshifts, whose uncertainties are large enough to preclude establishing a real physical association. Furthermore, in only three cases was the new companion galaxy at a smaller projected radius from the primary galaxy than the companion we had discovered within the MOSFIRE slit. Accordingly, we have demonstrated that our set of galaxy pairs is not missing a significant number of additional companions that fall outside the MOSFIRE slits but are at smaller separations from our primary galaxies than the companions identified within the MOSFIRE slits.

\section{Control Group Selection}

To establish the differential effects of merging on galaxy properties, we identified a control sample of spectroscopically confirmed galaxies within the MOSDEF survey that are characterized by similar galaxy properties (e.g., $M_{*}, z$ ) to those of our pairs, yet are confirmed to be lacking a physically associated companion galaxy. In other words, MOSDEF galaxies in the control sample are isolated. In assembling this control sample, we must bear in mind the fact that the MOSDEF pairs sample is highly incomplete. Indeed, given the small fraction of $360^{\circ}$ subtended by the MOSFIRE slit, there remains the possibility that a physically associated galaxy may be present within $60 \mathrm{kpc}$ (projected) of one of the MOSDEF primary targets (i.e., the separation that encompasses all of our MOSDEF pairs) but simply does not fall within the MOSFIRE slit. In such cases, the spectrum of the neighboring galaxy would not be collected, and this pair of galaxies would not be included in the MOSDEF pairs sample as defined in Section 3. To determine a sample of truly isolated galaxies within the set of MOSDEF primary targets, we considered each primary target in turn, searching through the 3D-HST photometry and redshift catalogs to identify possible neighbors that would not be flagged by our MOSFIRE-slit-based pairs criteria.

Based on the distribution of $R_{\text {proj }}$ in the MOSDEF pairs sample, we defined 60 projected $\mathrm{kpc}$ as the radius within which to search for companion galaxies around MOSDEF primary targets. In practice, we calculated the corresponding angular radius on the sky, given the redshift of the primary target. We then identified any possible companion galaxies in the 3D-HST survey whose coordinates placed them within this angular radius. For each possible companion, we estimated $\Delta z=\left|z_{\text {primary }}-z_{\text {companion }}\right|$, where $z_{\text {companion }}$ is the redshift of the potential companion galaxy. To determine if the apparent companion based on angular separation was also potentially associated with each primary target in redshift space, we 
Table 1

Observed Properties of MOSDEF Pairs

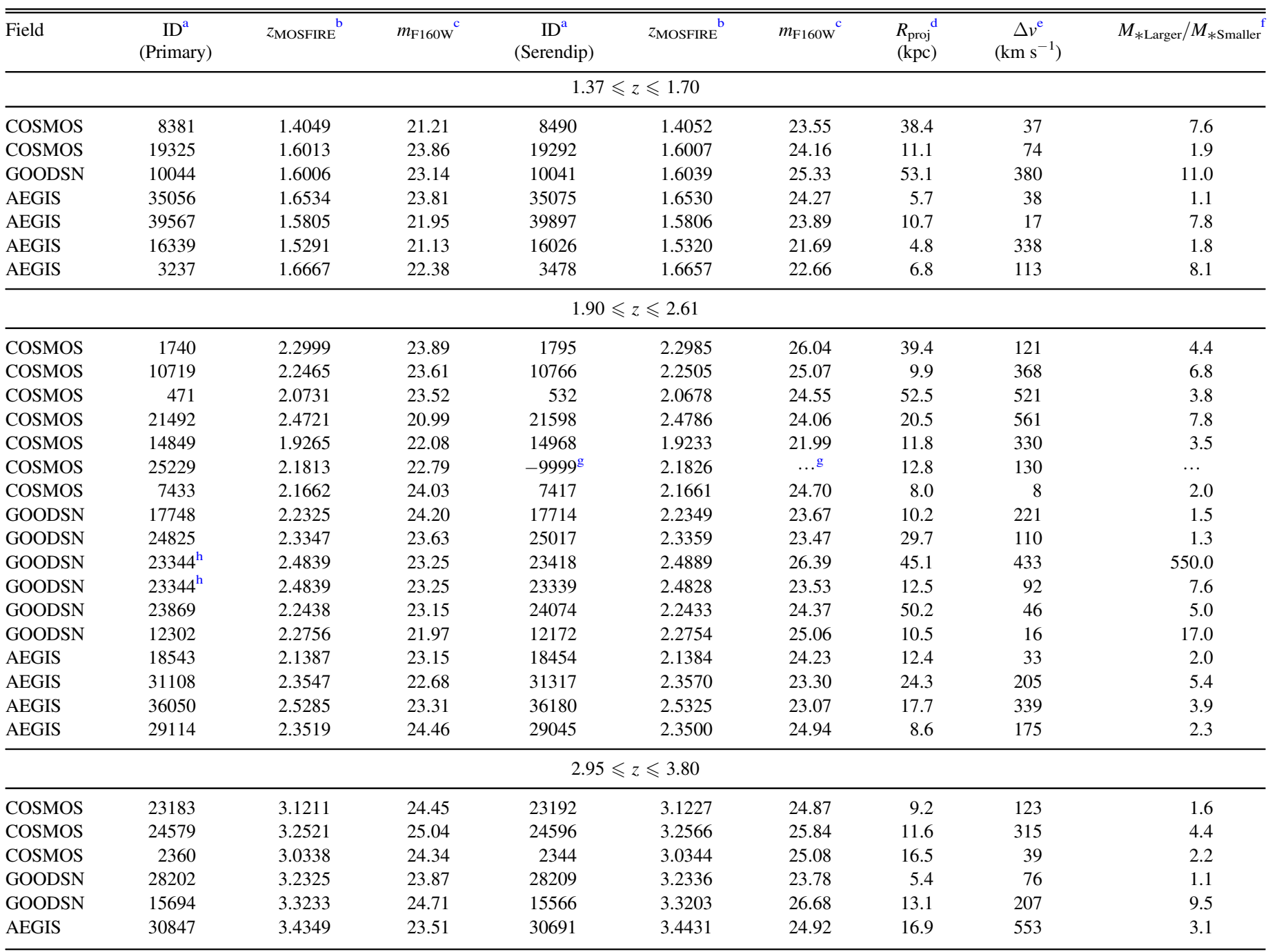

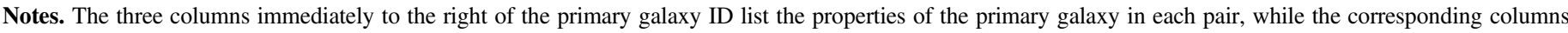
immediately to the right of the serendip ID refer to the serendip. The final two columns refer to the properties of the pair.

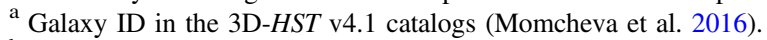

${ }^{\mathrm{b}}$ Emission-line redshift measured from MOSFIRE spectroscopy as part of the MOSDEF survey.

c HST/WFC3 F160W magnitude on the AB system.

${ }^{\mathrm{d}}$ Projected separation in kpc, based on the angular separation between the pair galaxies and the redshift of the primary galaxy.

e Velocity separation in $\mathrm{km} \mathrm{s}^{-1}$, based MOSFIRE systemic redshifts of the primary and serendip galaxies.

${ }^{\mathrm{f}}$ Stellar mass ratio between more passive and less massive pair member, irrespective of which galaxy is the primary and which is the serendip.

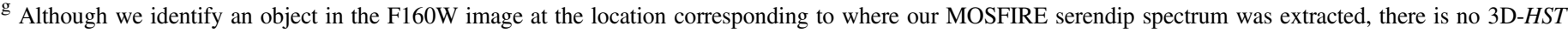

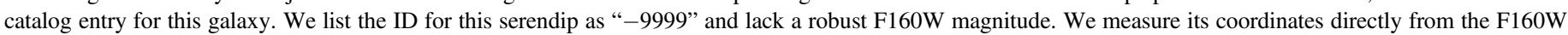
image, from which we determine the projected separation for this pair.

${ }^{\mathrm{h}}$ Primary galaxy with two serendip companions. The primary galaxy is listed twice, once for each serendip.

applied criteria that depended on the nature of the redshift measurement for the apparent companion. If the apparent companion had a previously measured spectroscopic redshift compiled by the 3D-HST survey (Brammer et al. 2012; Momcheva et al. 2016) or one measured in our own MOSDEF spectroscopy, we classified it as a true companion if $\left|z_{\text {primary }}-z_{\text {companion }}\right|<0.01$. If no spectroscopic redshift was available for the apparent companion, we used the 3D-HST grism redshifts $\left(z_{\text {grism }}\right)$. If no $z_{\text {grism }}$ was available, we used the 3D-HST photometric redshift, $z_{\text {phot }}$ (Momcheva et al. 2016). In the case of grism and photometric redshifts, we classified the apparent companion as a true companion if $z_{\text {primary }}$ was within the $68 \%$ confidence interval of the grism or photometric redshift probability distribution.

Accordingly, we identified isolated control galaxies as those that had no angular neighbors within $60 \mathrm{kpc}$ projected on the sky that were also true companions in redshift space (i.e., with $\left|z_{\text {primary }}-z_{\text {companion }}\right|<0.01$ for apparent companions with existing $z_{\text {spec }}$, or $z_{\text {primary }}$ within the $68 \%$ redshift confidence interval of apparent companions with existing $z_{\text {grism }}$ or $\left.z_{\text {phot }}\right)$. Using a more conservative isolation criterion for grism and photometric redshifts (i.e., forcing $z_{\text {primary }}$ to lie outside the $95 \%$ grism or photometric redshift confidence interval) yields a control sample that is more than two times smaller but 
Table 2

Physical Properties of MOSDEF Pairs

\begin{tabular}{|c|c|c|c|c|c|c|c|}
\hline \multirow{2}{*}{ Field } & \multirow{2}{*}{ ID } & \multirow{2}{*}{$\begin{array}{l}\text { Primary/ } \\
\text { Serendip }\end{array}$} & \multirow{2}{*}{$\begin{array}{c}\log \left(M_{*}\right)^{\mathrm{a}} \\
\left(M_{\odot}\right)\end{array}$} & \multirow{2}{*}{$\begin{array}{c}\log (\mathrm{SFR}(\mathrm{SED}))^{\mathrm{b}} \\
\left(M_{\odot} \mathrm{yr}^{-1}\right)\end{array}$} & \multirow{2}{*}{$\begin{array}{c}\log (\mathrm{SFR}(\mathrm{H} \alpha))^{\mathrm{c}} \\
\left(M_{\odot} \mathrm{yr}^{-1}\right)\end{array}$} & \multicolumn{2}{|c|}{$12+\log (\mathrm{O} / \mathrm{H})^{\mathrm{d}}$} \\
\hline & & & & & & $\overline{(\mathrm{O} 3 \mathrm{~N} 2)}$ & $\overline{(\mathrm{N} 2)}$ \\
\hline \multicolumn{8}{|c|}{$1.37 \leqslant z \leqslant 1.70$} \\
\hline $\operatorname{COSMOS}^{\mathrm{e}}$ & 8381 & primary & 10.94 & 0.60 & $\ldots$ & $<8.74$ & $<8.88$ \\
\hline COSMOS & 8490 & serendip & 10.06 & 0.00 & $\cdots$ & $\ldots$ & $\ldots$ \\
\hline COSMOS & 19325 & primary & 9.45 & 0.76 & 0.40 & $<8.46$ & $<8.52$ \\
\hline COSMOS & 19292 & serendip & 9.18 & 0.56 & $>0.91$ & $<8.61$ & $<9.00$ \\
\hline GOODSN & 10044 & primary & 9.79 & 0.83 & $>0.15$ & $\ldots$ & $<8.89$ \\
\hline GOODSN & 10041 & serendip & 8.75 & -0.21 & $>0.00$ & $<8.59$ & $<8.69$ \\
\hline AEGIS & 35056 & primary & 9.31 & 0.68 & $>1.21$ & $<8.14$ & $<8.29$ \\
\hline AEGIS & 35075 & serendip & 9.28 & 0.32 & 1.13 & $<8.14$ & $<8.32$ \\
\hline AEGIS & 39567 & primary & 10.39 & 1.43 & 1.96 & 8.36 & 8.44 \\
\hline AEGIS & 39897 & serendip & 9.50 & 0.83 & 0.70 & 8.33 & 8.46 \\
\hline AEGIS $^{\mathrm{e}}$ & 16339 & primary & 10.55 & 2.25 & 2.36 & 8.61 & 8.58 \\
\hline AEGIS $^{\text {e }}$ & 16026 & serendip & 10.83 & 1.87 & 1.70 & $\ldots$ & 8.71 \\
\hline AEGIS & 3237 & primary & 10.57 & 0.88 & $>1.15$ & $\cdots$ & 8.50 \\
\hline AEGIS & 3478 & serendip & 9.66 & 1.75 & 0.95 & 8.51 & 8.61 \\
\hline \multicolumn{8}{|c|}{$1.90 \leqslant z \leqslant 2.61$} \\
\hline COSMOS & 1740 & primary & 9.91 & 1.05 & 1.40 & 8.36 & 8.50 \\
\hline COSMOS & 1795 & serendip & 9.27 & -0.25 & $<1.20$ & $\cdots$ & $\cdots$ \\
\hline COSMOS & 10719 & primary & 11.04 & 0.88 & $\cdots$ & $\cdots$ & $\cdots$ \\
\hline COSMOS & 10766 & serendip & 10.21 & 0.69 & $>0.67$ & $\ldots$ & $<8.78$ \\
\hline $\operatorname{COSMOS}^{\mathrm{e}}$ & 471 & primary & 9.61 & 0.94 & $\ldots$ & $\ldots$ & 8.33 \\
\hline COSMOS & 532 & serendip & 9.03 & 1.55 & $\ldots$ & $\ldots$ & $<8.60$ \\
\hline $\operatorname{COSMOS}^{\mathrm{e}}$ & 21492 & primary & 11.06 & 2.39 & 2.21 & 8.39 & 8.63 \\
\hline COSMOS & 21598 & serendip & 10.17 & 0.94 & $>0.37$ & $<8.52$ & $<8.71$ \\
\hline COSMOS & 14849 & primary & 10.35 & 1.92 & $\ldots$ & $\ldots$ & $<8.76$ \\
\hline $\operatorname{cosMOS}^{\mathrm{e}}$ & 14968 & serendip & 9.80 & 1.97 & $\ldots$ & $\ldots$ & 8.70 \\
\hline COSMOS & 25229 & primary & 9.98 & 1.31 & 1.42 & 8.23 & 8.31 \\
\hline COSMOS & -9999 & serendip & $\ldots$ & $\ldots$ & $\ldots$ & $\ldots$ & $\cdots$ \\
\hline COSMOS & 7433 & primary & 9.70 & 0.84 & $>0.83$ & $<8.47$ & $<8.55$ \\
\hline COSMOS & 7417 & serendip & 9.40 & 0.79 & $>0.20$ & $<8.53$ & $<8.67$ \\
\hline GOODSN & 17748 & primary & 9.56 & 0.60 & 0.94 & $<8.25$ & $<8.33$ \\
\hline GOODSN & 17714 & serendip & 9.39 & 0.72 & 2.07 & $<8.07$ & $<8.17$ \\
\hline GOODSN & 24825 & primary & 9.75 & 0.43 & $\ldots$ & $\ldots$ & $\ldots$ \\
\hline GOODSN $^{\mathrm{e}}$ & 25017 & serendip & 9.65 & 0.70 & 1.36 & $\cdots$ & $\cdots$ \\
\hline GOODSN & 23344 & primary & 10.65 & 1.78 & 2.34 & 8.29 & 8.43 \\
\hline GOODSN & 23418 & serendip & 7.91 & 0.66 & $<1.37$ & $\ldots$ & $\ldots$ \\
\hline GOODSN & 23344 & primary & 10.65 & 1.78 & 2.34 & 8.29 & 8.43 \\
\hline GOODSN & 23339 & serendip & 9.77 & 1.33 & $<0.95$ & $\ldots$ & $\cdots$ \\
\hline GOODSN & 23869 & primary & 10.20 & 1.53 & 1.99 & 8.32 & 8.47 \\
\hline GOODSN & 24074 & serendip & 9.50 & 0.35 & $>0.45$ & $<8.41$ & $<8.59$ \\
\hline GOODSN $^{\mathrm{e}}$ & 12302 & primary & 10.92 & 2.11 & 1.99 & 8.56 & 8.68 \\
\hline GOODSN $^{\mathrm{e}}$ & 12172 & serendip & 9.69 & 0.32 & 1.40 & 8.36 & 8.45 \\
\hline AEGIS & 18543 & primary & 9.62 & 0.99 & 1.33 & 8.13 & 8.20 \\
\hline AEGIS & 18454 & serendip & 9.33 & 0.37 & $>1.99$ & $<8.35$ & $<8.58$ \\
\hline AEGIS & 31108 & primary & 10.73 & 1.91 & 2.17 & 8.36 & 8.47 \\
\hline AEGIS & 31317 & serendip & 10.00 & 0.68 & $\cdots$ & $\ldots$ & $\cdots$ \\
\hline AEGIS & 36050 & primary & 9.92 & 1.30 & 1.37 & 8.31 & 8.39 \\
\hline AEGIS & 36180 & serendip & 10.51 & 1.72 & 1.47 & 8.51 & 8.60 \\
\hline AEGIS & 29114 & primary & 9.32 & 0.36 & 0.62 & $<8.26$ & $<8.35$ \\
\hline AEGIS & 29045 & serendip & 8.95 & 0.51 & 0.24 & $<8.57$ & $<8.89$ \\
\hline \multicolumn{8}{|c|}{$2.95 \leqslant z \leqslant 3.80$} \\
\hline COSMOS & 23183 & primary & 10.06 & 1.82 & $\ldots$ & $\ldots$ & $\ldots$ \\
\hline COSMOS & 23192 & serendip & 10.27 & 1.60 & $\ldots$ & $\ldots$ & $\cdots$ \\
\hline COSMOS & 24579 & primary & 9.42 & 0.80 & $\ldots$ & $\ldots$ & $\ldots$ \\
\hline COSMOS & 24596 & serendip & 8.78 & 0.54 & $\ldots$ & $\ldots$ & $\ldots$ \\
\hline COSMOS & 2360 & primary & 9.73 & 1.06 & $\ldots$ & $\ldots$ & $\ldots$ \\
\hline COSMOS & 2344 & serendip & 9.39 & 0.90 & $\ldots$ & $\ldots$ & $\ldots$ \\
\hline GOODSN $^{\mathrm{e}}$ & 28202 & primary & 10.17 & 1.50 & $\cdots$ & $\ldots$ & $\ldots$ \\
\hline GOODSN & 28209 & serendip & 10.12 & 1.45 & $\ldots$ & $\ldots$ & $\ldots$ \\
\hline
\end{tabular}


Table 2

(Continued)

\begin{tabular}{|c|c|c|c|c|c|c|c|}
\hline \multirow[t]{2}{*}{ Field } & \multirow[t]{2}{*}{ ID } & \multirow{2}{*}{$\begin{array}{l}\text { Primary/ } \\
\text { Serendip }\end{array}$} & \multirow{2}{*}{$\begin{array}{c}\log \left(M_{*}\right)^{\mathrm{a}} \\
\left(M_{\odot}\right)\end{array}$} & \multirow{2}{*}{$\begin{array}{c}\log (\mathrm{SFR}(\mathrm{SED}))^{\mathrm{b}} \\
\left(M_{\odot} \mathrm{yr}^{-1}\right)\end{array}$} & \multirow{2}{*}{$\begin{array}{c}\log (\operatorname{SFR}(\mathrm{H} \alpha))^{\mathrm{c}} \\
\left(M_{\odot} \mathrm{yr}^{-1}\right)\end{array}$} & \multicolumn{2}{|c|}{$12+\log (\mathrm{O} / \mathrm{H})^{\mathrm{d}}$} \\
\hline & & & & & & $(\mathrm{O} 3 \mathrm{~N} 2)$ & $(\mathrm{N} 2)$ \\
\hline GOODSN & 15694 & primary & 9.81 & 1.14 & $\ldots$ & $\cdots$ & $\cdots$ \\
\hline GOODSN & 15566 & serendip & 8.83 & 0.34 & $\ldots$ & $\cdots$ & $\cdots$ \\
\hline AEGIS & 30847 & primary & 9.30 & 2.04 & $\ldots$ & $\ldots$ & $\cdots$ \\
\hline AEGIS & 30691 & serendip & 8.81 & 1.56 & $\ldots$ & $\ldots$ & $\ldots$ \\
\hline
\end{tabular}

Notes.

${ }^{\text {a }}$ Log stellar mass, in units of $M_{\odot}$.

${ }^{\mathrm{b}} \log$ of SFR derived from the best-fit population synthesis model, corrected for dust extinction, in units of $M_{\odot} \mathrm{yr}^{-1}$.

${ }^{c} \log$ of SFR derived from $\mathrm{H} \alpha$ luminosity, corrected for dust extinction, in units of $M_{\odot} \mathrm{yr}^{-1}$. An entry of “...” indicates galaxies lacking coverage of either $\mathrm{H} \alpha$ or $\mathrm{H} \beta$, or with limits in both $\mathrm{H} \alpha$ and $\mathrm{H} \beta$. SFRs for galaxies with $\mathrm{H} \alpha$ detections and $\mathrm{H} \beta$ non-detections are indicated as lower limits, while those for galaxies with $\mathrm{H} \alpha$ nondetections and $\mathrm{H} \beta$ detections are shown with upper limits.

${ }^{\mathrm{d}}$ Gas-phase oxygen abundance. The column labeled O3N2 lists oxygen abundances based on the O3N2 indicator, while the column labeled N2 contains oxygen abundances based on the N2 indicator (Pettini \& Pagel 2004). A value of “..." indicates galaxies lacking coverage of at least one of the required emission lines, or with enough non-detections to prevent derivation of a meaningful limit. Accordingly, we do not report oxygen abundances for galaxies in the highest-redshift bin.

${ }^{\mathrm{e}}$ Galaxy identified as an AGN based on X-ray, infrared, or rest-optical emission-line properties. Stellar mass, SFR, and metallicity values are not plotted or included in differential comparisons.

results that are statistically consistent with those presented in Section 5. Even with the $\sim 1 \sigma$ threshold in $\left|z_{\text {primary }}-z_{\text {companion }}\right|$ when $z_{\text {companion }}$ was based on a $z_{\text {grism }}$ or $z_{\text {phot }}$, we likely excluded a large number of isolated galaxies from the control sample that were not actually in physically associated pairs. Our requirements for isolation yield a sample of $372 \mathrm{MOSDEF}$ galaxies, 242 of which are at $1.4 \leqslant z \leqslant 2.6$, i.e., at redshifts where we can measure $\mathrm{H} \alpha$ and $[\mathrm{N}$ II] $\lambda 6584$, in addition to $\mathrm{H} \beta$ and $[\mathrm{O} \mathrm{III}] \lambda 5007$, and therefore obtain measurements of physical properties such as $\operatorname{SFR}(\mathrm{H} \alpha)$ and metallicity. These 242 galaxies in our control sample have a mean [median] redshift of $z=2.15 \pm 0.02[z=2.24 \pm 0.02]$ and a mean [median] stellar mass of $\log \left(M_{*} / M_{\odot}\right)=10.05 \pm 0.04$ $\left[\log \left(M_{*} / M_{\odot}\right)=9.92 \pm 0.04\right]$, as compared with a mean [median] redshift and stellar mass of $z=2.07 \pm 0.05[z=$ $2.23 \pm 0.06]$ and $\log \left(M_{*} / M_{\odot}\right)=9.85 \pm 0.10\left[\log \left(M_{*} / M_{\odot}\right)=\right.$ $9.77 \pm 0.07]$, respectively, for our pairs sample at $z<3$. When relevant in the following section, we consider a subset of the control sample that is even more precisely matched in median stellar mass to the pairs sample. Some local studies have also constructed control samples matched in environmental density (e.g., Ellison et al. 2010; Alonso et al. 2012). Given that we do not have robust environmental measures for the MOSDEF sample, we limit our matching to redshift and stellar mass. We also note that previous work has matched pair and control galaxies on a galaxy-by-galaxy basis (e.g., Ellison et al. 2008b; Scudder et al. 2012). In our analysis, however, our control sample of isolated galaxies is matched as a whole to have the same typical redshift and stellar mass as that of our significantly smaller pairs sample. In summary, the galaxies in our control sample lack physically associated companions and therefore constitute a powerful comparison data set alongside our sample of galaxy pairs within the same redshift range.

\section{Physical Properties of High-redshift Galaxy Pairs}

Studies of galaxy pairs in the local universe indicate measurable differences in their star formation activity and gas-phase oxygen abundances compared to isolated galaxies of similar masses. Enhancements in star formation are strongest at the smallest separations ( $\leqslant 20 \mathrm{kpc}$; Patton et al. 2013) and closest mass ratios (Ellison et al. 2008b; Scudder et al. 2012), but are detectable at greater than a factor of $\sim 1.3$ in galaxy pairs separated by up to 80 projected kpc (Scudder et al. 2012), characterized by mass ratios between 0.1 and 10 , and velocity differences $\Delta v<300 \mathrm{~km} \mathrm{~s}^{-1}$. Elevation in the SFRs of merging pairs relative to isolated galaxies has also been detected out to $z \sim 1$, both on average and also as a function of separation, with closer pairs at $0.5 \leqslant z \leqslant 1$ showing a greater SFR enhancement (Lin et al. 2007; Wong et al. 2011). To explore the impact of interactions on gas-phase oxygen abundance, Ellison et al. (2008b) construct the luminositymetallicity and mass-metallicity relation for both paired and isolated galaxies, demonstrating that galaxies in pairs are offset by $\sim 0.1$ dex toward lower gas-phase oxygen abundance at fixed luminosity and by $\sim 0.05$ dex toward lower metallicity at fixed stellar mass (see also Kewley et al. 2006; Scudder et al. 2012).

The observed differences between the star-forming and chemical abundance properties of galaxy pairs and their isolated counterparts have been explained using simulations of galaxy interactions. In Patton et al. (2013), it is shown that simulations of merging galaxies yield SFR enhancements even out to pair separations of $\sim 150 \mathrm{kpc}$, due to star formation triggered by a preceding close passage. As for the observed metallicity differences in galaxy pairs, it is seen that tidal forces and gravitational torques present during mergers can lead to inflows of low-metallicity gas into the merging galaxy nuclei and a corresponding dilution in oxygen abundance (Torrey et al. 2012, 2019; Bustamante et al. 2018).

We used our MOSDEF pairs and control samples to investigate if differences in SFR and metallicity can be detected in early-stage mergers relative to isolated galaxies at $1.4 \leqslant z \leqslant 2$.6. In Section 5.1, we consider the star-forming main sequence of pairs and control objects, while, in Section 5.2 we explore potential differences in the MZR.

\subsection{The $\mathrm{SFR}-\mathrm{M}_{*}$ Relation}

To investigate trends in SFR for interacting and isolated galaxies at high redshift, we assembled SFR and $M_{*}$ values for both our pairs sample and the corresponding control sample 

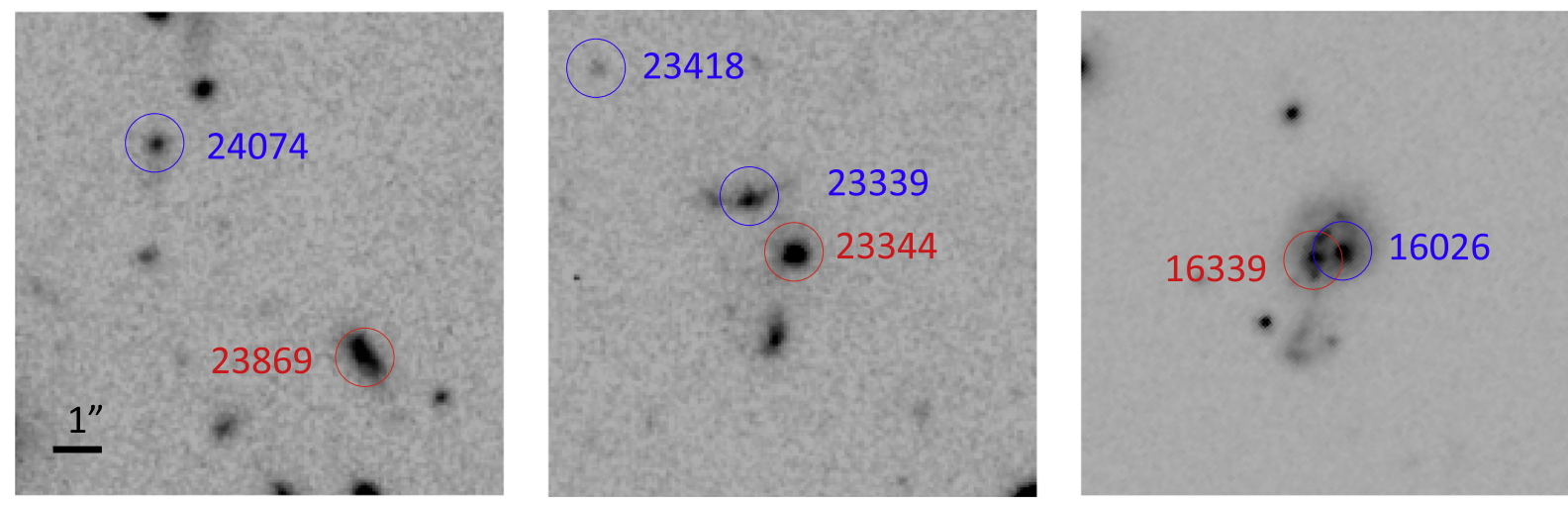

Figure 4. $H S T$ WFC3/F160W postage stamps for pairs in our sample, demonstrating the variety of systems covered. Each postage stamp is $10^{\prime \prime} \times 10^{\prime \prime}$, oriented with north up and east to the left. In each image, the primary target is indicated in red, while the serendips are labeled in blue. Further details of each merging pair are provided in Tables 1 and 2. Left: a wide-separation pair, consisting of GOODSN-23869 (primary; $z=2.2438$ ) and GOODSN-24074 (serendip; $z=2.2433$ ). Center: A triple of associated galaxies, including GOODSN-23344 (primary; $z=2.4839$ ), GOODSN-23339 (serendip; $z=2.4828$ ), and GOODSN-23418 (serendip; $z=2.4889$ ). The galaxy just to the south of GOODSN-23344 (i.e., GOODSN-23271) does not have a spectroscopic redshift, but its photometric redshift is consistent with the spectroscopic redshift of GOODSN-23344. Right: one of the pairs with the smallest observed separation, suggesting coalescence. This pair consists of AEGIS-16339 (primary; $z=1.5291$ ) and AEGIS-16026 (serendip; $z=1.5320$ ). This system also shows qualitative evidence for extended tidal features.

over the redshift range $1.4 \leqslant z \leqslant 2.6$. For this analysis, we considered SFRs derived from dust-corrected $\mathrm{H} \alpha$ and $\mathrm{H} \beta$ emission lines $(\operatorname{SFR}(\mathrm{H} \alpha))$, and also from the same fits to broadband SEDs that yielded estimates of $M_{*}$ (SFR(SED)). Prior to comparing the pairs and control samples, we removed any objects flagged as AGNs based on X-ray, IR, or opticalemission-line properties ${ }^{12}$ (Coil et al. 2015; Azadi et al. 2017) as in such cases both the Balmer emission-line fluxes and the Spitzer/IRAC photometric points included in the SED fitting may be contaminated by radiation associated with the AGN rather than star formation.

We show the distributions of SFR and $M_{*}$ for both pairs and control samples in Figure 6, displaying SFR(SED) and SFR $(\mathrm{H} \alpha)$, respectively, in the left- and right-hand panels. Only $\sim 50 \%$ of the pairs and control samples in the target redshift range have $\operatorname{SFR}(\mathrm{H} \alpha)$ detections. The majority of the remaining galaxies have only limits in $\operatorname{SFR}(\mathrm{H} \alpha)$ because of $\mathrm{H} \beta$ or $\mathrm{H} \alpha$ non-detections. For the rest (not plotted), we have no constraints on $\operatorname{SFR}(\mathrm{H} \alpha)$ because neither $\mathrm{H} \alpha$ nor $\mathrm{H} \beta$ is detected, or else we lack coverage for either or both $\mathrm{H} \alpha$ and $\mathrm{H} \beta$. Given the sample incompleteness, it is not straightforward to make quantitative inferences regarding the relative distributions of $\operatorname{SFR}(\mathrm{H} \alpha)$ and $M_{*}$ for our pairs and control samples. However, we note that the distributions of $\operatorname{SFR}(\mathrm{H} \alpha)$ detections and limits for the pairs and control samples are qualitatively similar.

Both Reddy et al. (2015) and Shivaei et al. (2016) have shown that there is general agreement between SFR(SED) and $\operatorname{SFR}(\mathrm{H} \alpha)$ values for galaxies in the MOSDEF sample. Therefore, to draw more quantitative conclusions regarding the SFR versus $M_{*}$ distributions of interacting and isolated systems, we use measurements of SFR(SED) versus $M_{*}$. We have such measurements for all galaxies in our pairs and control samples (with the exception of the unidentified serendip associated with COSMOS-25229), and therefore incompleteness is not an issue. For a quantitative comparison, we perform an ordinary least-squares regression fit to both the pairs and control samples. For the regression

\footnotetext{
${ }^{12}$ For this analysis, we used a simple criterion of $\log (\mathrm{N}$ II $\lambda 6584 / \mathrm{H} \alpha)>-0.3$ to flag an object as an AGN. We did not apply this criterion to galaxies at $2.95 \leqslant z \leqslant 3.80$ because their spectra lacked coverage of $\mathrm{H} \alpha$ and [N II] $\lambda 6584$.
}

analysis, we restrict the samples to $\log \left(M_{*} / M_{\odot}\right)>8,{ }^{13}$ and exclude quiescent galaxies obviously offset from the distribution of star-forming systems (i.e., we do not include galaxies with $\log \left(\mathrm{SFR}(\mathrm{SED}) / M_{\odot} \mathrm{yr}^{-1}\right) \leqslant-1$ or those with $\log \left(M_{*} / M_{\odot}\right)>11$ and $\left.\log \left(\mathrm{SFR}(\mathrm{SED}) / M_{\odot} \mathrm{yr}^{-1}\right) \leqslant 0.2\right)$. To obtain error estimates on the best-fit intercept and slope, we perturb individual $M_{*} / M_{\odot}$ and SFR(SED) values according to their errors 1000 times and refit the perturbed data sets. The upper and lower confidence bounds we report span $68.3 \%$ of the fits to the perturbed data sets. We find for the pairs:

$$
\begin{aligned}
\log (\mathrm{SFR}(\mathrm{SED}))_{\text {pairs }}= & -4.98_{-0.90}^{+1.30} \\
& +0.60_{-0.13}^{+0.09} \times \log \left(M_{*} / M_{\odot}\right)_{\text {pairs }}
\end{aligned}
$$

and for the control sample:

$$
\begin{aligned}
\log (\operatorname{SFR}(\mathrm{SED}))_{\text {control }}= & -3.80_{-0.20}^{+0.45} \\
& +0.49_{-0.05}^{+0.02} \times \log \left(M_{*} / M_{\odot}\right)_{\text {control }} .
\end{aligned}
$$

Accordingly, the best-fit regressions for pairs and control galaxies are consistent with each other, indicating no elevation in star formation at fixed mass for galaxies in pairs relative to isolated systems. If anything, galaxies in pairs are offset toward slightly lower SFR(SED) at fixed $M_{*}$, but this offset is not significant. This result holds when we consider only pairs at separations of $R_{\text {proj }} \leqslant 30 \mathrm{kpc}$; i.e., the separation at which measurable enhancement appears in the local pairs sample in Ellison et al. (2008b). For the $R_{\text {proj }} \leqslant 30 \mathrm{kpc}$ pair sample, we find very similar best-fit regression parameters to those for the full pairs sample:

$$
\begin{aligned}
\log (\operatorname{SFR}(\mathrm{SED}))_{\text {pairs }}= & -4.75_{-0.90}^{+1.40} \\
& +0.58_{-0.15}^{+0.10} \times \log \left(M_{*} / M_{\odot}\right)_{\text {pairs }} .
\end{aligned}
$$

We note that the slope and intercept values reported here for the relationship between SFR(SED) versus $M_{*}$ differ from

\footnotetext{
13 Accordingly, we do not include the low-mass companion object GOODSN23418, which is a factor of 550 lower in mass than its corresponding primary galaxy, GOODSN-23344, and consider a sample with mass ratios spanning a range comparable to that Ellison et al. (2008b).
} 


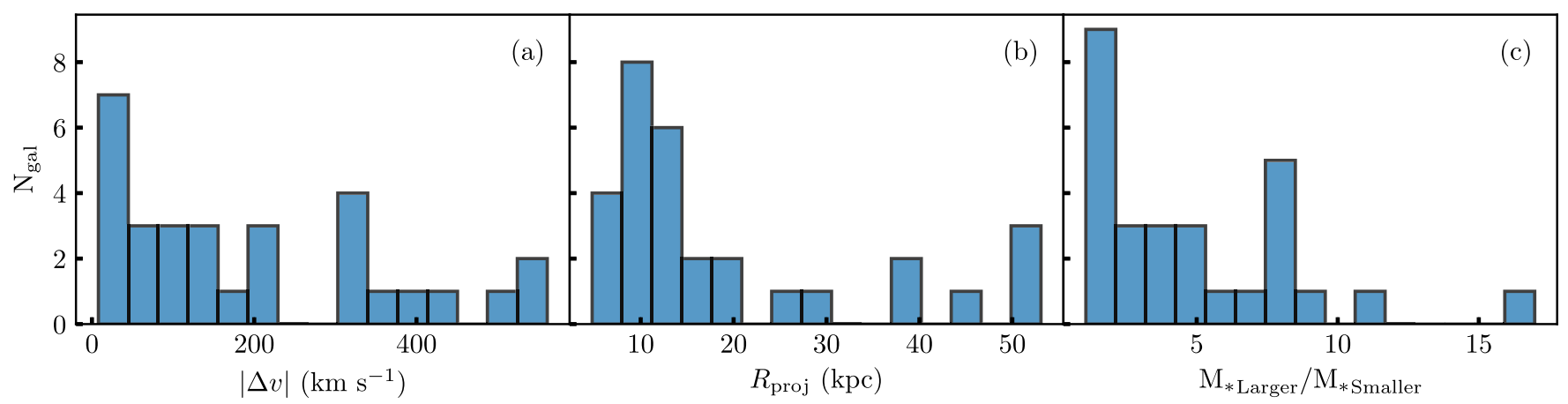

Figure 5. (a) Histogram of line of sight velocity difference, $|\Delta v|$ between primary and serendip objects. (b) Histogram of projected physical separation $R_{\text {proj }}$ between primary and serendip objects. (c) Histogram of stellar mass ratio between more massive and less massive objects, irrespective of which galaxy is the primary and which is the serendip. For improved display quality, we exclude from this panel the one pair with a mass ratio of 550, zooming on the pairs ranging in mass ratio between 1.1 and 17 .

those in Shivaei et al. (2015) (see Table 1 of that paper) and Sanders et al. (2018), mainly due to differences in the methodology adopted for the regression analysis. However, given that our goal here is to make a direct comparison between pairs and control galaxies, and because we use identical regression methodology for those two samples (which we do), our inferences about their respective SFR(SED) versus $M_{*}$ relations are robust.

As another way to quantify the star-forming properties of pairs and control galaxies, we estimate the median SFR(SED) for the pairs sample used in the regression analysis, and for a subset of the control sample used for regression analysis that is matched precisely in median stellar mass (i.e., $\left.\log \left(M_{*} / M_{\odot}\right)_{\text {med }}=9.75\right)$. We find $\log (\operatorname{SFR}(\mathrm{SED}))_{\text {pairs,med }}=$ $0.83 \pm 0.09$ for the full sample of pairs, and $\log (\operatorname{SFR}(\operatorname{SED}))_{\text {pairs,med }}=0.84 \pm 0.09$ for the subsample with $R_{\text {proj }} \leqslant 30 \mathrm{kpc}$. For the control sample matched in median stellar mass, we find $\log (\mathrm{SFR}(\mathrm{SED}))_{\text {control,med }}=0.92 \pm 0.04$. The difference between pair and control sample medians is $\Delta \mathrm{SFR}_{\text {med }}=-0.09 \pm 0.10$ for the full pairs sample and $\triangle S F R_{\text {med }}=-0.08 \pm 0.11$ for the pairs sample with $R_{\text {proj }} \leqslant 30 \mathrm{kpc}$. Based on these results, we do not detect a significant difference in the sample median SFR(SED) values for pairs and control galaxies. If anything, the pairs sample is offset toward lower median SFR(SED) (but not significantly).

To compare with results based on galaxy pairs in SDSS, we make use of the catalog presented in (Patton et al. 2013, 2016; S. L. Ellison et al. 2018, private communication). We identify a local sample of 830 galaxies in pairs with $\Delta v \leqslant 500 \mathrm{~km} \mathrm{~s}^{-1}$, $R_{\text {proj }} \leqslant 30 \mathrm{kpc}$, and stellar mass ratios closer than 10:1, and a local control sample of 17,835 galaxies having no companions within $\Delta v=500 \mathrm{~km} \mathrm{~s}^{-1}$ or $R_{\text {proj }}=150 \mathrm{kpc}$. In this catalog, SFR is defined as that contained within the SDSS fiber and is based on dust-corrected $\mathrm{H} \alpha$ emission. The SDSS pairs and control samples are well matched overall in median and mean stellar mass and redshift, but the median SFR of the pairs sample is elevated by $\Delta \mathrm{SFR}_{\text {med }}=0.16 \pm 0.03$. Comparing the results for MOSDEF and SDSS pairs with $R_{\text {proj }} \leqslant 30 \mathrm{kpc}$ and $\Delta v \leqslant 500 \mathrm{~km} \mathrm{~s}^{-1}\left(\Delta \mathrm{SFR}_{\text {med }}=-0.08 \pm 0.11\right.$ for MOSDEF pairs and $\triangle S_{F R}$ med $=0.16 \pm 0.03$ for SDSS pairs), we find a difference in the SFR enhancement measured at high and low redshift at the $\sim 2 \sigma$ level. A larger sample of high-redshift pairs will reduce the uncertainty on the median properties and enable more definitive conclusions.
In addition to considering the global star-forming properties of our pairs sample, we also investigate the properties of galaxies in pairs with small mass ratios $(\leqslant 3: 1)$ and those with pair separations smaller than $10 \mathrm{kpc}$, checking whether such galaxies in particular show enhanced star formation relative to the control sample. We measure the residuals in SFR(SED) for these two subsamples of objects around the best-fit linear regression to the full pairs sample (i.e., $\log (\mathrm{SFR}(\mathrm{SED}))-$ $\log \left(\operatorname{SFR}(\mathrm{SED})_{\mathrm{fit}}\right.$, where $\log \left(\mathrm{SFR}(\mathrm{SED})_{\mathrm{fit}}\right.$ is taken from Equation (3)), and find no significant vertical offset on average for either the small mass ratio or small separation subsample. Although our high-redshift pairs sample is small, with the associated uncertainties of a small sample size, our results differ qualitatively from the elevation in star formation at fixed mass and the dependence of elevated star formation on mass ratio and pair separation detected for lower-redshift interacting galaxies (e.g., Lin et al. 2007; Ellison et al. 2008b; Wong et al. 2011; Patton et al. 2013). We discuss these differences further in Section 6.

One final caveat about the results in this section is that the SFR(SED) values we derive are not sensitive to star formation that is completely obscured at rest-UV and optical wavelengths (Chapman et al. 2005). Therefore, if a higher fraction of the star formation in galaxy pairs is highly obscured, we will miss it and miss potential differences in the total bolometric SFRs of pairs and control galaxies. Deeper far-infrared data are needed to address this concern.

\subsection{The MZR Relation}

We also analyzed the distribution of pairs and control galaxies in the space of metallicity and mass, considering the MZR for these two samples. In this analysis, we used two different metallicity indicators-N2 and O3N2 (Pettini \& Pagel 2004) - to check whether the results depended on the method for estimating oxygen abundance. We again restricted this analysis to the redshift range $1.4 \leqslant z \leqslant 2.6$, over which $\mathrm{H} \beta$, [O III] $\lambda 5007, \mathrm{H} \alpha$ and [N II] $\lambda 6584$ emission lines are all accessible in ground-based spectroscopy, and removed AGNs as in Section 5.1. We show the distributions of $12+\log (\mathrm{O} / \mathrm{H})$ and $M_{*}$ in Figure 7 for both pairs and control galaxies. As in the case of $\operatorname{SFR}(\mathrm{H} \alpha)$, our galaxy samples have roughly equal numbers of detections and limits, mainly due to non-detections in $\mathrm{H} \beta$ or $[\mathrm{N} \mathrm{II}] \lambda 6584$. 

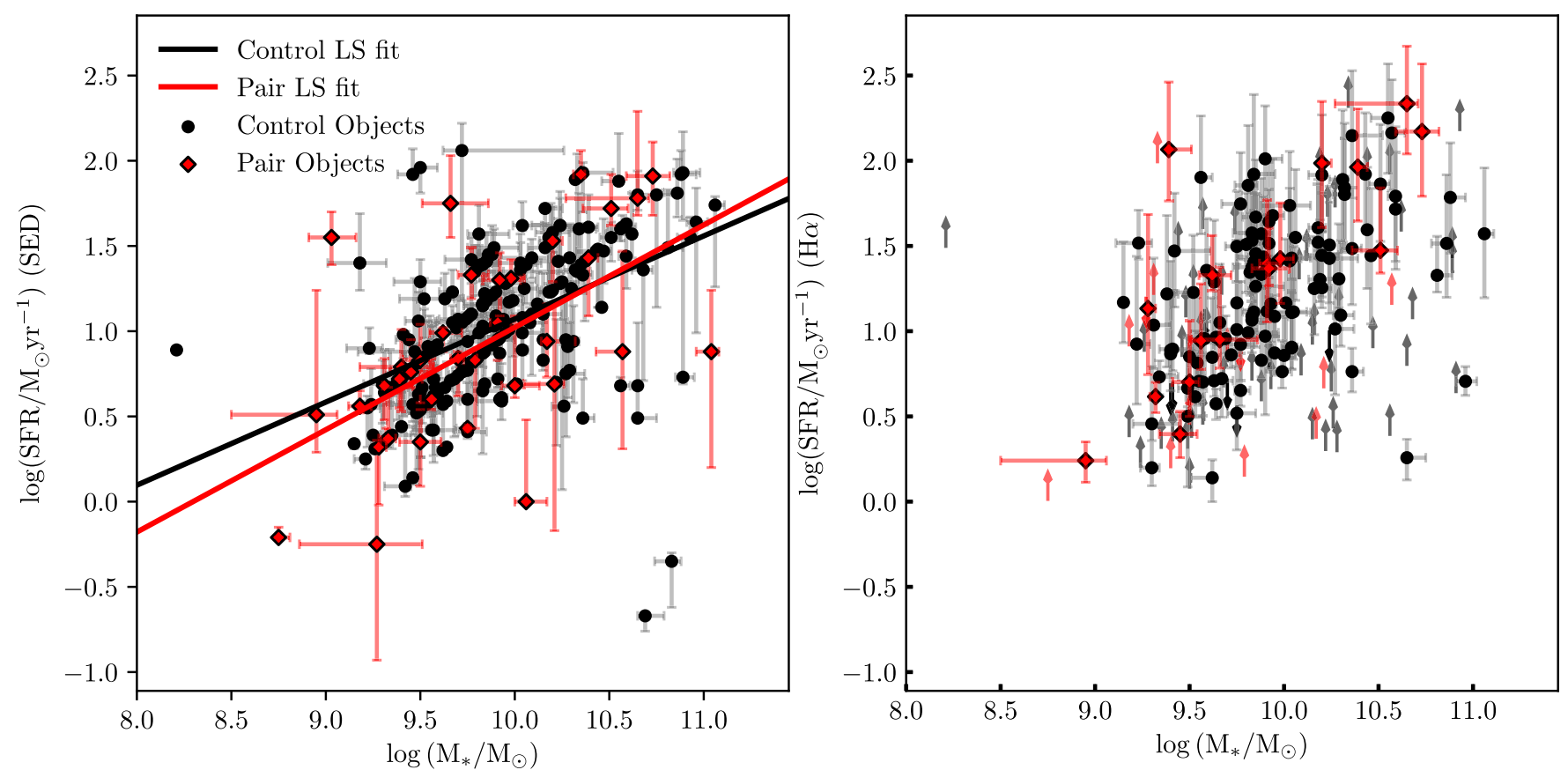

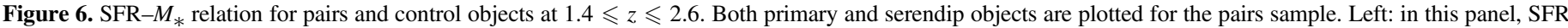

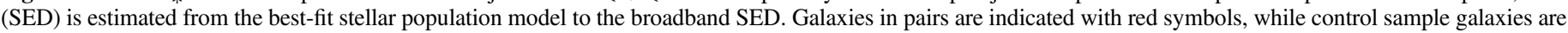

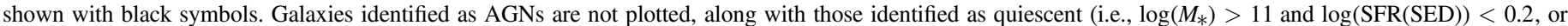

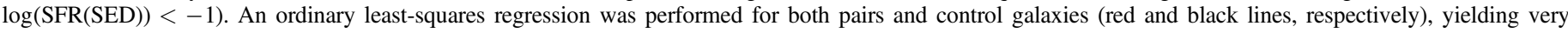

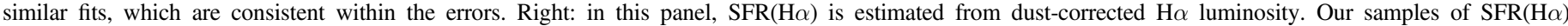

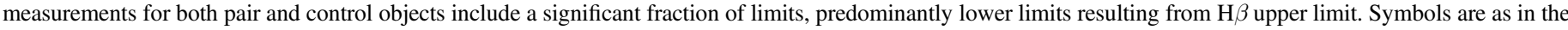

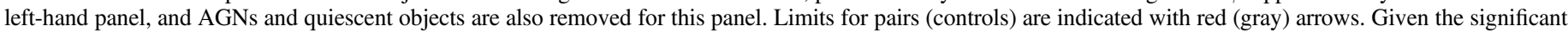

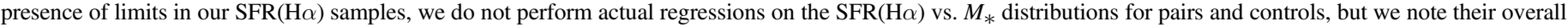
similarity.

To quantify the average trends in mass and metallicity, and incorporate information from galaxies with individual limits, we construct median composite spectra in two bins of stellar mass for both pairs and control samples, following the methodology described in Sanders et al. (2018). The only requirements for inclusion in these stacks are coverage of $\mathrm{H} \alpha$ and $[\mathrm{N} \mathrm{II}] \lambda 6584$ in the case of the $\mathrm{N} 2$ stacks, additional coverage of $[\mathrm{O} \mathrm{III}] \lambda 5007$ and $\mathrm{H} \beta$ for the $\mathrm{O} 3 \mathrm{~N} 2$ stacks, and a detection of $\mathrm{H} \alpha$ emission at $\geqslant 3 \sigma$. Accordingly, the sample of objects included in the O3N2 stacks is a subset of the objects in the N2 stacks, given the additional requirement of [O III] $\lambda 5007$ and $\mathrm{H} \beta$ coverage. For composite measurements of [N II] $\lambda 6584 / \mathrm{H} \alpha$, we used $H$-band spectra for galaxies at $1.4 \leqslant$ $z \leqslant 1.7$, and $K$-band spectra for those at $1.9 \leqslant z \leqslant 2.7$. For the corresponding measurements of $[\mathrm{O} \mathrm{III}] \lambda 5007 / \mathrm{H} \beta$, we used $J$-band spectra for galaxies at $1.4 \leqslant z \leqslant 1.7$ and $H$-band spectra for those at $1.9 \leqslant z \leqslant 2.7 . \mathrm{H} \alpha$ and $\mathrm{H} \beta$ emission-line measurements from the composite spectra were corrected for underlying stellar Balmer absorption based on the median absorption strength for individual galaxies going into the stack, as estimated from stellar population synthesis models to broadband photometry. Dust extinction corrections were not applied to the composite spectra, given the close proximity of [N II] $\lambda 6584$ and $\mathrm{H} \alpha$, and that of [O III] $\lambda 5007$ and $\mathrm{H} \beta$. The median $M_{*}$ in each stellar mass bin and $12+\log (\mathrm{O} / \mathrm{H})$ estimated from the corresponding composite spectrum are are shown in Figure 7, along with the individual measurements and limits. It is clear that the pairs and control galaxies follow very similar trends in $12+\log (\mathrm{O} / \mathrm{H})$, regardless of whether $\mathrm{N} 2$ or $\mathrm{O} 3 \mathrm{~N} 2$ is considered. The same results hold when we consider the subset of MOSDEF pairs at $R_{\text {proj }} \leqslant 30 \mathrm{kpc}$. We recall here that local pairs show a depression in metallicity at fixed luminosity and stellar mass relative to isolated systems (e.g., Ellison et al. 2008b; Scudder et al. 2012) in apparent contrast to our own preliminary high-redshift results. However, the uncertainty on the metallicities estimated from the pairs composite spectra is equal to or larger than the observed $z \sim 0$ depression in metallicity $(\sim 0.05 \mathrm{dex})$. We therefore cannot rule out a $\leqslant 0.05$ dex offset in metallicity between interacting pairs and isolated galaxies at fixed $M_{*}$.

\section{Discussion and Future Directions}

We have demonstrated that merging galaxy pairs at $1.4 \leqslant z \leqslant 2.6$ are not characterized by elevated SFRs or significantly diluted metallicities relative to isolated systems of the same stellar mass. Although we will require a larger pairs sample to place these results on a more secure statistical footing, it is worthwhile to consider the implications of the suggested trends, which run contrary to the patterns uncovered among interacting galaxies at lower redshift. As we discuss in this section, there is a theoretical basis for the apparent evolution in the differential star-forming properties of merging systems. Here we compare our results with other differential studies of merging pairs at $z>1$, consider the predictions from simulations, and discuss evidence for AGN activity in our merging systems. We conclude by listing some promising future directions for the study of interacting galaxies at high redshift.

\subsection{Comparisons with Other Observational Work}

Our study represents the first controlled differential comparison of SFRs in interacting and isolated galaxies at $z>1$. 


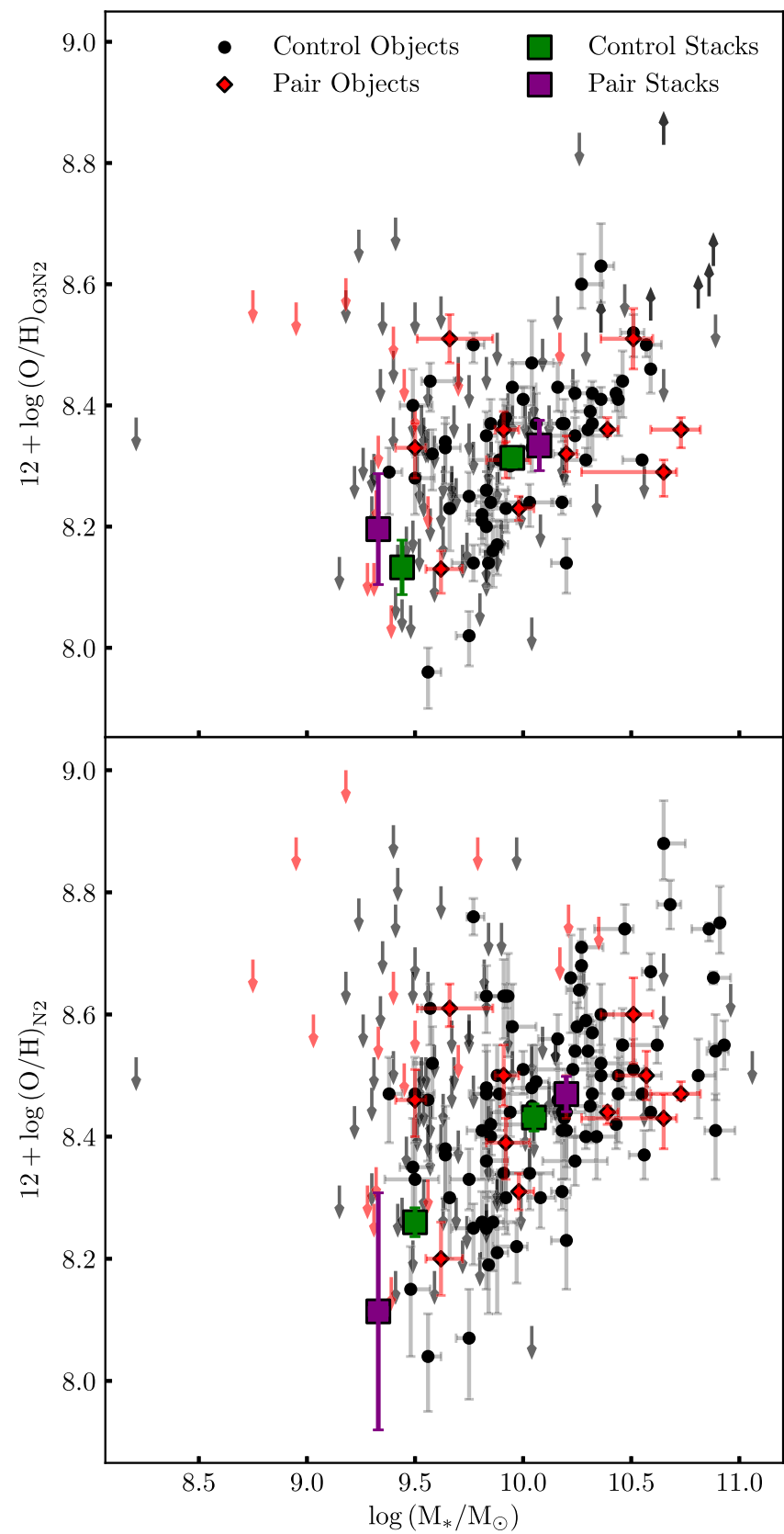

Figure 7. MZR for pairs and control objects at $1.4 \leqslant z \leqslant 2.6$. Top: in this panel, $12+\log (\mathrm{O} / \mathrm{H})$ is determined from the $\mathrm{O} 3 \mathrm{~N} 2$ indicator and the calibration of Pettini \& Pagel (2004). Galaxies in pairs are shown with red symbols, while those in the control sample are indicated in black. Metallicity limits are indicated as red (gray) arrows for pairs (controls). To include information from individual non-detections, we constructed median composite spectra for pairs and control galaxies, in two bins of $M_{*}$ for each sample. We then measured emission-line ratios from the composite spectra in each stellar mass bin, and the corresponding O3N2 metallicities. Metallicities from the stacked spectra are shown in purple for the pairs sample and in green for the control sample. These stacked measurements indicate no evidence for dilution in metallicity at fixed $M_{*}$ for galaxies in the pairs sample. Bottom: in this panel, $12+\log (\mathrm{O} / \mathrm{H})$ is determined from the N2 indicator and the calibration of Pettini \& Pagel (2004). Symbols are as in the top panel. Measurements from N2 composite spectra also indicate no evidence for metallicity dilution in interacting systems.

However, Divoy et al. (2014) previously considered the question of the relationship between metallicity and smallscale environment. These authors analyzed a sample of 49 starforming galaxies at $z \sim 0.9-1.8$ with VLT/SINFONI integral field unit emission-line maps and associated N2 metallicity measurements. In this sample, 12 systems are identified as interacting, based on the presence of a companion within $\Delta v=500 \mathrm{~km} \mathrm{~s}^{-1}$ and $R_{\text {proj }}=30 h^{-1} \mathrm{kpc}$ (López-Sanjuan et al. 2013). Divoy et al. (2014) find a measurable depression in median metallicity of at least $0.13 \mathrm{dex}$ for their interacting sample relative to a control sample of 37 isolated galaxies at similar median stellar mass. This depression is significantly larger than what is observed in the local universe $(\leqslant 0.05 \mathrm{dex}$; Ellison et al. 2008b; Scudder et al. 2012), and is in contrast to the lack of significant offset in metallicity that we find for our pairs relative to the control sample. To obtain a more robust result at high redshift, we require a significantly larger sample of galaxy pairs with metallicity measurements and deeper spectroscopy sufficient to detect $\mathrm{H} \beta$ and $[\mathrm{N} \mathrm{II}] \lambda 6584$ for a larger fraction of the sample to reduce the number of metallicity upper limits.

\subsection{Expectations from Simulations}

The lack of enhancement in SFR observed for the MOSDEF pairs sample is consistent with recent predictions from numerical simulations of galaxy formation. Fensch et al. (2017) ran a suite of pc-scale galaxy merger simulations, representing low-redshift galaxies with gas fractions of $10 \%$, while $z=2$ galaxies were simulated with gas fractions of $60 \%$. For the same orbital parameters, it is found that the gas-poor merger simulations approximating local galaxies feature boosts in the SFR of the merging galaxies of an order of magnitude or more over an extended period of hundreds of Myr prior to coalescence. Meanwhile, the gas-rich simulations approximating $z=2$ show only mild increases in SFR, and only at coalescence. Based on a suite of binary galaxy merger simulations with identical orbital parameters but nine different initial gas fractions ranging from $M_{\text {gas }} / M_{*}=0.04$ to 1.78 , Scudder et al. (2015) similarly find that the enhancement in SFR during the merger is anti-correlated with initial gas fraction. Fensch et al. (2017) attribute the difference in the evolution of the SFR during the merger to differences in the increase of both central gas inflows and compressive turbulence in the ISM during mergers at $z=2$ and $z=0$. Both gas-rich $(z=2)$ and gas-poor $(z=0)$ merger simulations reach similar peak central gas inflow rates fueling star formation. However, given that the gas-rich simulations start off with premerger baseline gas inflow rates that are an order of magnitude higher than those in the gas-poor simulations, the enhancement in gas inflow and corresponding SFR is significantly weaker. Fensch et al. (2017) then attribute the lack of increase in ISM turbulence during $z=2$ mergers to both an ISM velocity dispersion that is higher premerger and harder to additionally stir-up (Wisnioski et al. 2015), and a clumpy ISM architecture with an associated tidal field that also suppresses an increase in turbulence (Genzel et al. 2008). These simulations do not incorporate cosmological accretion or the more compact nature of $z=2$ galaxies relative to those at $z=0$ of the same mass, although the authors argue that differences in gas fraction and ISM structure are the dominant effects underlying the observed difference in SFR evolution for high-redshift mergers. Analysis of the lower-resolution Horizon-AGN cosmological hydrodynamical simulation by Martin et al. (2017) also predicts that the enhancement in SFR at fixed mass due to mergers is most pronounced at low redshift and undetectable at $z>1.5$, when 
the ambient premerger level of star formation is an order of magnitude higher than in the local universe.

There is also recent theoretical work focusing on the evolution of ISM metallicity during galaxy mergers. Based on a sample of 70 gas-rich mergers traced at $z<1.5$ in the Auriga cosmological simulation, Bustamante et al. (2018) find that a period of metallicity dilution typically occurs during merger events, reaching a magnitude of $\Delta Z=-0.1$ dex for major mergers at projected separations $<10 \mathrm{kpc}$ and at least a few hundredths of a dex depression for both major and minor mergers at separations of $<30 \mathrm{kpc}$. These results are roughly consistent with observations of $z \sim 0$ merging pairs by, e.g., Scudder et al. (2012). However, it is also worth noting that the precoalescence depressions in metallicity recovered in these simulations are consistent with those inferred from the fundamental metallicity relation (FMR; Ellison et al. 2008a; Mannucci et al. 2010). Specifically, given the relation among $M_{*}$, SFR, and gas-phase oxygen abundance in the FMR, the metallicity during the mergers is consistent with predictions from the FMR, given the evolution in SFR and $M_{*}$ of the merging galaxies. Accordingly, given that our merging pairs show no offset from the control sample in the SFR versus $M_{*}$ relation, and given that these pairs represent a precoalescence phase, the expectation of the simulation results of Bustamante et al. (2018) is that pairs in MOSDEF should simply follow the same relationship among $M_{*}$, SFR, and metallicity that is inferred at $z \sim 2$ for the MOSDEF sample as a whole (Sanders et al. 2018). Furthermore, given the size of the error bars on our median stacked metallicities, we are not sensitive to differences of $\leqslant 0.05$ dex as observed by Ellison et al. (2008b) and Scudder et al. (2012), and predicted for all but the most extreme merger events in Bustamante et al. (2018).

Finally, we note that Torrey et al. (2019) have also found evidence for metallicity dilution during mergers in the IllustrisTNG cosmological simulation, but the detailed example analyzed in that work consisted of a merger at $z \sim 0$. It is not clear how the analogous results would differ at $z \sim 2$. Highresolution " $z=2$ " simulations, such as those of Fensch et al. (2017), are required to address the question of metallicity dilution in high-redshift mergers. For robust conclusions, such simulations must also include cosmological gas accretion, and track metal enrichment as well as star formation.

\subsection{The AGN Fraction in MOSDEF Pairs}

Our samples of galaxies in pairs are indistinguishable from their isolated counterparts in terms of star formation and metallicity at fixed stellar mass. The extensive multi-wavelength data available for MOSDEF targets also enables AGN classifications on the basis of X-ray luminosity, infrared colors, and/or rest-optical emission-line ratios (Coil et al. 2015; Azadi et al. 2017). Given the connection between galaxy mergers and black hole fueling inferred from numerical simulations (e.g., Di Matteo et al. 2005; Hopkins et al. 2006), much observational work has been devoted to exploring the link between galaxy interactions and AGN activity, with mixed results. For example, Ellison et al. (2011) demonstrate an enhancement in AGN fraction among galaxy close pairs at $z \sim 0$, with up to a factor of 2.5 enhancement in AGN fraction for pairs with projected separations less than $40 \mathrm{kpc}$. Along the same lines, Goulding et al. (2018) use machine-learning classifications of merging systems at $z \sim 0-0.9$, finding an AGN fraction (as estimated by WISE infrared colors) elevated by a factor of $\sim 2-7$ among interacting relative to non-interacting systems. Many other investigations have focused instead on the merger fraction among AGNs (as opposed to the AGN fraction among mergers), finding that AGNs do not show a significantly enhanced fraction of mergers relative to their inactive counterparts (e.g., Gabor et al. 2009; Kocevski et al. 2012; Hewlett et al. 2017). Exploring the merger fraction among AGNs and non-AGNs provides constraints on how important interactions are for driving AGN activity relative to other more secular processes (Bournaud et al. 2011). Given the design of our experiment and the incompleteness of our pairs sample (see Section 4), we are not in a position to quantify the differential merger fraction among AGNs and non-AGNs. However, we can obtain complete estimates of the AGN fraction among merging and isolated galaxies, which indicates how likely it is for mergers to trigger AGN activity.

Based on the X-ray, infrared, and rest-optical emission-line AGN classifications for MOSDEF galaxies, we find that in our full pairs sample, the fraction of galaxies satisfying at least one of these AGN criteria is 10 out of $59(16.9 \% \pm 5.3 \%$, with the error based on simple Poisson statistics). Focusing on the $1.4 \leqslant z \leqslant 2.6$ subsample, for which we compared SFRs and metallicities with their isolated counterparts, we find that 9 out of $47(19.1 \% \pm 6.3 \%)$ are classified as AGNs. We also note that 7 out of $36(19.4 \% \pm 7.3 \%)$ of these systems with $R_{\text {proj }} \leqslant 30 \mathrm{kpc}$-i.e., the same fraction-satisfy at least one of the AGN criteria. The corresponding AGN fractions for the full and $1.4 \leqslant z \leqslant 2.6$ control subsamples are 42 out of 372 $(11.3 \% \pm 1.7 \%)$ and 32 out of $242(13.2 \% \pm 2.3 \%)$. Accordingly, we find a higher AGN fraction among pairs relative to controls, with a factor of at least $\sim 1.5$ enhancement in the pair AGN fraction at $1.4 \leqslant z \leqslant 2.6$. However, given the small sample size of the pairs, this difference is not highly significant. Increasing the sample size of pairs and control galaxies by an order of magnitude will enable robust statistics on the differential AGN fraction between pairs and control galaxies.

\subsection{Future Work}

Our analysis comprises an early step in characterizing the properties of merging galaxies at high redshift. With only 30 pairs in total, 24 of which we analyze in detail along with a carefully defined control sample of isolated galaxies, the statistical power of our sample is limited. For example, while Ellison et al. (2008b) and Scudder et al. (2012) divided their samples of almost 2000 merging pairs into multiple bins of projected separation and mass ratio to explore how the physical properties of merging galaxies depend on each of these merger characteristics, our current small sample size precludes such division. In addition to obtaining a significantly larger sample of merging pairs, we must also obtain significantly deeper rest-optical emission-line spectra, so that the fraction of limits in $\operatorname{SFR}(\mathrm{H} \alpha)$ and $12+\log (\mathrm{O} / \mathrm{H})$ is reduced to a negligible contribution across a sufficiently wide mass range. Accordingly, we will be able to analyze the distributions of individual merging and isolated galaxies in the spaces of $\operatorname{SFR}(\mathrm{H} \alpha)$ versus $M_{*}$ and $12+\log (\mathrm{O} / \mathrm{H})$ versus $M_{*}$.

We also need to apply an effective technique or identifying later-stage mergers closer to coalescence, when the effects of SFR enhancement and metallicity dilution are predicted to be strongest (Bustamante et al. 2018). At low redshift, nonparametric morphological measures are commonly used to identify such mergers (Lotz et al. 2004), but Abruzzo et al. (2018) 
demonstrate with mock observations of cosmological simulations that these same merger morphological classifications at $z \sim 2$ are significantly contaminated by non-merging galaxies and should not be applied to HST images of high-redshift galaxies. Another route to studying later-stage mergers would consist of building up a significantly larger (i.e., two orders of magnitude) sample of spectroscopically confirmed pairs with $R_{\text {proj }}<10 \mathrm{kpc}$ pairs and $\Delta v \leqslant 500 \mathrm{~km} \mathrm{~s}^{-1}$. With larger galaxy pairs samples, accompanied by complete sets of emission-line detections and a larger range of merger stages, we will truly be able to test models of galaxy mergers in the early universe.

We thank the referee for an extremely constructive and thorough report. We are deeply grateful to Sara Ellison and David Patton for sharing their SDSS catalog of local starforming galaxies and their closest companions. We acknowledge support from NSF AAG grants AST-1312780, 1312547, 1312764, and 1313171, archival grant AR-13907 provided by NASA through the Space Telescope Science Institute, and grant NNX16AF54G from the NASA ADAP program. This work was supported by the NSF under NSF REU grant No. PHY-1460055, along with support from the Department of Physics \& Astronomy of the University of California, Los Angeles. R.L.S. was supported by a UCLA Graduate Division Dissertation Year Fellowship. We also acknowledge a NASA contract supporting the "WFIRST Extragalactic Potential Observations (EXPO) Science Investigation Team" (15WFIRST15-0004), administered by GSFC. We also acknowledge the 3D-HST collaboration, who provided us with spectroscopic and photometric catalogs used to select MOSDEF targets and derive stellar population parameters. We wish to extend special thanks to those of Hawaiian ancestry on whose sacred mountain we are privileged to be guests. Without their generous hospitality, most of the observations presented herein would not have been possible.

\section{ORCID iDs}

Alice E. Shapley (1) https://orcid.org/0000-0003-3509-4855 Ryan L. Sanders (1) https://orcid.org/0000-0003-4792-9119 Naveen A. Reddy (i) https://orcid.org/0000-0001-9687-4973 William R. Freeman (1) https://orcid.org/0000-00033559-5270

Mariska Kriek (i) https://orcid.org/0000-0002-7613-9872

Irene Shivaei iㅏ https://orcid.org/0000-0003-4702-7561

Alison L. Coil @i https://orcid.org/0000-0002-2583-5894

Brian Siana (1) https://orcid.org/0000-0002-4935-9511

Sedona H. Price (ㅈ) https://orcid.org/0000-0002-0108-4176

Mojegan Azadi (1) https://orcid.org/0000-0001-6004-9728

Guillermo Barro (ib https://orcid.org/0000-0001-6813-875X

Laura de Groot (i) https://orcid.org/0000-0001-9022-665X

Francesca M. Fornasini (i) https://orcid.org/0000-0002-

9286-9963

Gene C. K. Leung (i) https://orcid.org/0000-0002-9393-6507

\section{References}

Abruzzo, M. W., Narayanan, D., Davé, R., \& Thompson, R. 2018, MNRAS, submitted (arXiv:1803.02374)

Alonso, S., Mesa, V., Padilla, N., \& Lambas, D. G. 2012, A\&A, 539, A46

Azadi, M., Coil, A. L., Aird, J., et al. 2017, ApJ, 835, 27

Bluck, A. F. L., Conselice, C. J., Bouwens, R. J., et al. 2009, MNRAS, 394, L51

Bournaud, F., Dekel, A., Teyssier, R., et al. 2011, ApJL, 741, L33
Brammer, G. B., van Dokkum, P. G., Franx, M., et al. 2012, ApJS, 200, 13 Bustamante, S., Sparre, M., Springel, V., \& Grand, R. J. J. 2018, MNRAS, 479, 3381

Calzetti, D., Armus, L., Bohlin, R. C., et al. 2000, ApJ, 533, 682

Cardelli, J. A., Clayton, G. C., \& Mathis, J. S. 1989, ApJ, 345, 245

Chabrier, G. 2003, PASP, 115, 763

Chapman, S. C., Blain, A. W., Smail, I., \& Ivison, R. J. 2005, ApJ, 622, 772

Coil, A. L., Aird, J., Reddy, N., et al. 2015, ApJ, 801, 35

Conroy, C., Gunn, J. E., \& White, M. 2009, ApJ, 699, 486

Conselice, C. J. 2014, ARA\&A, 52, 291

Cox, T. J., Jonsson, P., Somerville, R. S., Primack, J. R., \& Dekel, A. 2008, MNRAS, 384, 386

Di Matteo, T., Springel, V., \& Hernquist, L. 2005, Natur, 433, 604

Divoy, C., Contini, T., Pérez-Montero, E., et al. 2014, A\&A, 569, A64

Ellison, S. L., Patton, D. R., Mendel, J. T., \& Scudder, J. M. 2011, MNRAS, 418, 2043

Ellison, S. L., Patton, D. R., Simard, L., et al. 2010, MNRAS, 407, 1514

Ellison, S. L., Patton, D. R., Simard, L., \& McConnachie, A. W. 2008a, ApJL, 672, L107

Ellison, S. L., Patton, D. R., Simard, L., \& McConnachie, A. W. 2008b, AJ, 135,1877

Fensch, J., Renaud, F., Bournaud, F., et al. 2017, MNRAS, 465, 1934

Freeman, W. R., Siana, B., Kriek, M., et al. 2017, arXiv:1710.03230

Gabor, J. M., Impey, C. D., Jahnke, K., et al. 2009, ApJ, 691, 705

Genzel, R., Burkert, A., Bouché, N., et al. 2008, ApJ, 687, 59

Goulding, A. D., Greene, J. E., Bezanson, R., et al. 2018, PASJ, 70, S37 Grogin, N. A., Kocevski, D. D., Faber, S. M., et al. 2011, ApJS, 197, 35

Hao, C.-N., Kennicutt, R. C., Johnson, B. D., et al. 2011, ApJ, 741, 124

Hewlett, T., Villforth, C., Wild, V., et al. 2017, MNRAS, 470, 755

Hopkins, P. F., Croton, D., Bundy, K., et al. 2010, ApJ, 724, 915

Hopkins, P. F., Hernquist, L., Cox, T. J., et al. 2006, ApJS, 163, 1

Hopkins, P. F., Hernquist, L., Cox, T. J., \& Kereš, D. 2008, ApJS, 175, 356

Kennicutt, R. C., Jr. 1998, ARA\&A, 36, 189

Kewley, L. J., Geller, M. J., \& Barton, E. J. 2006, AJ, 131, 2004

Kocevski, D. D., Faber, S. M., Mozena, M., et al. 2012, ApJ, 744, 148

Koekemoer, A. M., Faber, S. M., Ferguson, H. C., et al. 2011, ApJS, 197, 36

Kriek, M., Shapley, A. E., Reddy, N. A., et al. 2015, ApJS, 218, 15

Kriek, M., van Dokkum, P. G., Labbé, I., et al. 2009, ApJ, 700, 221

Lin, L., Koo, D. C., Weiner, B. J., et al. 2007, ApJL, 660, L51

Lofthouse, E. K., Kaviraj, S., Conselice, C. J., Mortlock, A., \& Hartley, W. 2017, MNRAS, 465, 2895

López-Sanjuan, C., Le Fèvre, O., Tasca, L. A. M., et al. 2013, A\&A, 553, A78 Lotz, J. M., Jonsson, P., Cox, T. J., et al. 2011, ApJ, 742, 103

Lotz, J. M., Primack, J., \& Madau, P. 2004, AJ, 128, 163

Madau, P., \& Dickinson, M. 2014, ARA\&A, 52, 415

Man, A. W. S., Toft, S., Zirm, A. W., Wuyts, S., \& van der Wel, A. 2012, ApJ, 744,85

Man, A. W. S., Zirm, A. W., \& Toft, S. 2016, ApJ, 830, 89

Mannucci, F., Cresci, G., Maiolino, R., Marconi, A., \& Gnerucci, A. 2010, MNRAS, 408, 2115

Mantha, K. B., McIntosh, D. H., Brennan, R., et al. 2018, MNRAS, 475, 1549 Martin, G., Kaviraj, S., Devriendt, J. E. G., et al. 2017, MNRAS, 472, L50

McLean, I. S., Steidel, C. C., Epps, H. W., et al. 2012, Proc. SPIE, 8446, $84460 \mathrm{~J}$

Mihos, J. C., \& Hernquist, L. 1996, ApJ, 464, 641

Momcheva, I. G., Brammer, G. B., van Dokkum, P. G., et al. 2016, ApJS, 225, 27

Moreno, J., Bluck, A. F. L., Ellison, S. L., et al. 2013, MNRAS, 436, 1765

Patton, D. R., Ellison, S. L., Simard, L., McConnachie, A. W., \& Mendel, J. T. 2011, MNRAS, 412, 591

Patton, D. R., Qamar, F. D., Ellison, S. L., et al. 2016, MNRAS, 461, 2589

Patton, D. R., Torrey, P., Ellison, S. L., Mendel, J. T., \& Scudder, J. M. 2013, MNRAS, 433, L59

Pettini, M., \& Pagel, B. E. J. 2004, MNRAS, 348, L59

Pettini, M., Shapley, A. E., Steidel, C. C., et al. 2001, ApJ, 554, 981

Reddy, N. A., Kriek, M., Shapley, A. E., et al. 2015, ApJ, 806, 259

Sanders, D. B., \& Mirabel, I. F. 1996, ARA\&A, 34, 749

Sanders, R. L., Shapley, A. E., Kriek, M., et al. 2015, ApJ, 799, 138

Sanders, R. L., Shapley, A. E., Kriek, M., et al. 2018, ApJ, 858, 99

Scudder, J. M., Ellison, S. L., Momjian, E., et al. 2015, MNRAS, 449, 3719

Scudder, J. M., Ellison, S. L., Torrey, P., Patton, D. R., \& Mendel, J. T. 2012, MNRAS, 426, 549

Shapley, A. E., Steidel, C. C., Pettini, M., \& Adelberger, K. L. 2003, ApJ, 588,65

Shivaei, I., Kriek, M., Reddy, N. A., et al. 2016, ApJL, 820, L23

Shivaei, I., Reddy, N. A., Shapley, A. E., et al. 2015, ApJ, 815, 98 
Skelton, R. E., Whitaker, K. E., Momcheva, I. G., et al. 2014, ApJS, 214, 24 Steidel, C. C., Erb, D. K., Shapley, A. E., et al. 2010, ApJ, 717, 289 Steidel, C. C., Rudie, G. C., Strom, A. L., et al. 2014, ApJ, 795, 165 Tacconi, L. J., Genzel, R., Lutz, D., et al. 2002, ApJ, 580, 73

Tasca, L. A. M., Le Fèvre, O., López-Sanjuan, C., et al. 2014, A\&A, 565, A10 Torrey, P., Cox, T. J., Kewley, L., \& Hernquist, L. 2012, ApJ, 746, 108
Torrey, P., Vogelsberger, M., Marinacci, F., et al. 2019, MNRAS, 484, 5587 Ventou, E., Contini, T., Bouché, N., et al. 2017, A\&A, 608, A9

Williams, R. J., Quadri, R. F., \& Franx, M. 2011, ApJL, 738, L25

Wisnioski, E., Förster Schreiber, N. M., Wuyts, S., et al. 2015, ApJ, 799, 209

Wong, K. C., Blanton, M. R., Burles, S. M., et al. 2011, ApJ, 728, 119 